PsyArXiv

\title{
Why Overfitting is Not (Usually) a Problem in Partial Correlation Networks
}

\author{
Donald R. Williams \& Josue E. Rodriguez \\ University of California, Davis
}

\begin{abstract}
Network psychometrics is undergoing a time of methodological reflection. In part, this was spurred by the revelation that $\ell_{1}$-regularization does not reduce spurious associations in partial correlation networks. In this work, we address another motivation for the widespread use of regularized estimation: the thought that it is needed to mitigate overfitting. We first clarify important aspects of overfitting and the bias-variance tradeoff that are especially relevant for the network literature, where the number of nodes or items in a psychometric scale are not large compared to the number of observations (i.e., a low $p / n$ ratio). This revealed that bias and especially variance are most problematic in $p / n$ ratios rarely encountered. We then introduce a nonregularized method, based on classical hypothesis testing, that fulfills two desiderata: (1) reducing or controlling the false positives rate and (2) quelling concerns of overfitting by providing accurate predictions. These were the primary motivations for initially adopting the graphical lasso (glasso). In several simulation studies, our nonregularized method provided more than competitive predictive performance, and, in many cases, outperformed glasso. It appears to be nonregularized, as opposed to regularized estimation, that best satisfies these desiderata. We then provide insights into using our methodology. Here we discuss the multiple comparisons problem in relation to prediction: stringent alpha levels, resulting in a sparse network, can deteriorate predictive accuracy. We end by emphasizing key advantages of our approach that make it ideal for both inference and prediction in network analysis.
\end{abstract}

Keywords: partial correlation network, overfitting, prediction, frequentist inference, mean squared error

In the social-behavioral sciences, network theory has emerged as an increasingly popular framework for understanding psychological constructs (Borsboom, 2017; Jones, Heeren, \& McNally, 2017). The underlying rationale is that a group of observed variables, say, self-reported symptoms, are a dynamic system that mutually influence and interact with one another (Borsboom \& Cramer, 2013). The observed variables are "nodes" and the featured connections between nodes are "edges." This work focuses on partial correlation networks, wherein the edges represent conditionally dependent nodes-pairwise relations that have controlled for the other nodes in the network (Epskamp, Waldorp, Mottus, \& Borsboom, 2018). This powerful approach has resulted in an explosion of research; for example, network analysis has been used to shed new light upon a variety of constructs including personality (Costantini et al., 2015), narcissism (Di Pierro, Costantini, Benzi, Madeddu, \& Preti, 2019), and

DRW was supported by a National Science Foundation Graduate Research Fellowship under Grant No. 1650042. All code to reproduce the simulations and figures is available online (https://osf.io/fm92b/). hypersexuality (Werner, Štulhofer, Waldorp, \& Jurin, 2018).

Recently, the foundation of network psychometrics was improved upon when the default methodology was revisited (Williams \& Rast, 2019; Williams, Rhemtulla, Wysocki, $\&$ Rast, 2019). In the network literature, $\ell_{1}$-regularization (a.k.a., "least absolute shrinkage and selection operator" or "lasso") emerged as the default approach for detecting conditionally dependent relations. Initially, it was motivated by the thought that it reduces spurious relations. Paradoxically, the exact opposite holds true: lasso is known to not select the correct model (Zhao \& Yu, 2006) and to have a relatively low false negative rate. For the latter, in structural equations models (SEM), it was noted that "lasso kept more variables in the model (more Type I and fewer Type II errors)" (p. 72, Jacobucci, Brandmaier, \& Kievit, 2019). In network models, it was recently demonstrated that the inflated false positive rate inherent to lasso depends on many factors, including the sample size, edge size, sparsity, and the number of nodes (see Figure 6 in Williams et al., 2019). On the other hand, nonregularized estimation has a lower false positive rate that does not depend on those factors (Williams \& Rast, 2019). Together, it is now clear that limiting false positives does not motivate the default status of $\ell_{1}$-regularization.

In this work, we seek to further improve network anal- 
ysis by revisiting another purported benefit of using $\ell_{1}$ regularization. Additional motivation for using lasso is the thought that it can mitigate overfitting. The underlying rationale is summarized in Fried and Cramer (2017):

In the case of network models, overfitting is an especially severe challenge because we investigate relationships among a large number of variables, which means there is danger of overfitting a large number of parameters. One way to mitigate this problem somewhat is to regularize networks (p. 1011).

Although Fried and Cramer (2017) also stated "it is unclear at present to what degree regularization techniques increase generalizability" (p. 1011), it has nonetheless permeated the network literature. Indeed, the emerging consensus is that $\ell_{1}$-regularization provides "protection against overfitting" ( $\mathrm{p}$. 16, Christensen \& Golino, 2019). We argue that such statements are not entirely clear, as, for example, it is all but guaranteed that the "fit to the data" (measured by predictive accuracy) will be better for training data than unseen datasome degree of overfitting is a foregone conclusion. In our opinion, it is also not readily apparent that overfitting is a "severe challenge" that motivates regularization compared to nonregularized estimation. Because regularization has serious ramifications on statistical inference, such as presenting issues for computing valid confidence intervals (see for example section 3.1 in Bühlmann, Kalisch, \& Meier, 2014), the guiding idea behind this work is that the "challenge" of overfitting must warrant sacrificing gold-standard statistical approaches (e.g., ordinary least squares and $p$-values).

The network literature provides a unique opportunity to investigate predictive methods in the social-behavioral sciences. This is because nodewise predictability has an important place in both network theory and analysis (Haslbeck \& Fried, 2017; Haslbeck \& Waldorp, 2018). The basic idea is to see how well a given node is predicted by the other nodes in the network. The primary motivation of this approach is to determine "how self-determined the network is" (p. 860, Haslbeck \& Waldorp, 2018). This is accomplished by computing variance explained for each node in the network, given the corresponding "neighborhood" of conditional relations (i.e., shared connections with other nodes, Meinshausen \& Bühlmann, 2006). This approach is used extensively in the network literature, including as an outcome measure in clinical interventions (see for example Blanken et al., 2019). Therefore, it is important to develop methodology that brings to fruition the primary motivations for initially adopting $\ell_{1}$ regularization: (1) detecting conditional relations with a low false positive rate; and (2) mitigating the possibility of overfitting. As we show below, both goals can be achieved without employing regularization.

At first blush it may sound naive to suggest nonregularized methods can not only provide adequate but actually superior predictive performance compared to $\ell_{1}$-regularization. However, in certain situations, this is a robust finding in the model selection literature (see references in Bertsimas, King, \& Mazumder, 2016; Mazumder, Radchenko, \& Dedieu, 2017). This is summarized in Hastie, Tibshirani, and Tibshirani (2017):

The lasso gives better accuracy results than [nonregularized] best subset selection in the low signal-to-noise ratio (SNR) range and worse accuracy than best subset in the high SNR range. The transition point- the SNR level past which best subset outperforms the lasso-varies depending on the problem dimensions (p. 17).

The transition point refers to the number of variables $(p)$ relative to the number of observations $(n)$. Hence, even in noisy situations, the benefits of lasso diminish or vanish altogether when $n$ is much larger than $p$. This is the customary situation in the network literature. The remaining consideration is the SNR (i.e., $\frac{R^{2}}{1-R^{2}}$ ). Nodes are usually items from a validated psychometric scale, that, by construction, should not have a terribly low SNR. Together, this suggests that the oft-touted protective shield of regularization may be overstated.

The thought that regularization is advantageous for limiting overfitting extends beyond the network literature. In the social-behavioral sciences, this is typically presented with some combination of regularization and the bias-variance tradeoff (Jacobucci et al., 2019; Yarkoni \& Westfall, 2017). It is often argued that biasing estimates with regularization can be advantageous, for example, "[lasso] introduces bias in the parameter estimates in order to avoid overfitting"( $p$. 6, Ryan, Bringmann, \& Schuurman, 2019). However, it is important to note that parameter and prediction bias are not the same thing. Indeed, from the statistics literature, "the nonzero estimates from the lasso tend to be biased toward zero, so the debiasing [removing parameter bias]...can often improve the prediction error of the model" (p. 16, Hastie, Tibshirani, \& Wainwright, 2015). In addition to proposing novel methodology, another major contribution of this work is clarifying possible confusion surrounding the biasvariance tradeoff and overfitting.

\section{Major Contribution}

Our major contribution is explicitly examining overfitting in partial correlation networks. In the literature, the general thought is that overfitting is a serious challenge that requires using regularization. However, as noted in Waldorp, Marsman, and Maris (2019), "Once the parameters are obtained it turns out that inference on network parameters is in general difficult with $\ell_{1}$-regularization" (p. 53). Accordingly, because inference is the primary goal in network, overfitting must be a pressing challenge that warrants sacrificing statistical inference. If not, this would indicate that there 
is no reason to use regularization, given that "inference on network parameters" is straightforward with non-regularized methods.

This contribution is similar in spirit to Williams and Rast (2019) and Williams et al. (2019), both of which demonstrated that $\ell_{1}$-regularization has a relatively high false positive rate. In this work, however, the focus is explicitly on the purported benefits of $\ell_{1}$-regularization for overfitting in network analysis.

\section{Overview}

In what follows, we first introduce partial correlation networks and predictability in the context of a motivating example. Here we bring much needed nuance and clarity to customary examples, including the infamous polynomial regression and the bias-variance tradeoff, that are used to discuss regularization. To this end, we transport ideas stemming from econometrics and machine learning to the psychological literature. Second, we introduce a rather simple, nonregularized method for both detecting conditional relations with a low false positive rate and predictive accuracy. We then compare this method to $\ell_{1}$-regularization using real datasets that span a wide range of psychological constructs. We conclude with recommendations for computing nodewise predictability in psychological networks.

\section{The Gaussian Graphical Model}

For multivariate normal data, the Gaussian graphical model (GGM) captures conditional relationships that are typically visualized to infer the underlying dependence structure (i.e., the partial correlation "network"; Højsgaard, Edwards, \& Lauritzen, 2012; Lauritzen, 1996). There is an undirected graph that is denoted $G=(V, E)$, which includes a vertex set $V=\{1, \ldots, p\}$ and an edge set $E \subset V \times V$. The former refers to "nodes" and the set represents, say, items in a questionnaire, whereas the latter set contains the estimated network structure. Let $\mathbf{y}=\left(y_{1}, \ldots, y_{p}\right)^{\top}$ be a random vector indexed by the graph's vertices that is assumed to follow a multivariate normal distribution, $\mathbf{y} \sim \mathcal{N}_{p}(\boldsymbol{\mu}, \boldsymbol{\Sigma})$, where $\boldsymbol{\Sigma}$ is a $p \times p$ positive definite covariance matrix. We use $\mathbf{Y}$ to denote the $n \times p$ data matrix, where each row corresponds to the observations from a given individual. Further, without loss of information, the data are considered centered with mean vector 0 . The undirected graph is obtained by determining which off-diagonal elements of the precision matrix, $\boldsymbol{\Theta}=\boldsymbol{\Sigma}^{-1}$, are non-zero. That is, $(i, j) \in E$ when node $i$ and $j$ are determined to be conditionally dependent and set to zero otherwise. Note that the edges (or "connections") in a GGM are partial correlations $\rho_{i j \cdot z}$ that are computed directly from $\boldsymbol{\Theta}$, that is,

$$
\rho_{i j \cdot z}=\frac{-\theta_{i j}}{\sqrt{\theta_{i i} \theta_{j j}}}
$$

Hence, estimating partial correlation networks can be accomplished by testing whether each relation in (1) is "significantly" different from zero. This is described in Drton and Perlman (2004) and Williams and Rast (2019). The method introduced below builds upon this general idea.

\section{Multiple Regression}

Network predictability takes advantage of the correspondence between the elements of $\boldsymbol{\Theta}$ and multiple regression (Kwan, 2014; Stephens, 1998). Suppose that the jth column $\mathbf{Y}_{j}$ is predicted by the remaining $(p-1)$ nodes $\mathbf{Y}_{-j}$. For nodes $i$ and $j$, the resulting coefficients and error variances are defined as

$$
\beta_{i j}=\frac{-\theta_{i j}}{\theta_{i i}} \text { and } \sigma_{j}^{2}=\frac{1}{\theta_{i i}},
$$

where $i$ and $j$ denote the corresponding row and column of $\boldsymbol{\Theta}, \beta_{i j}$ is the regression weight for the $j$ th node $(i \neq j)$, and $\sigma_{j}^{2}$ is the residual variance. This allows for recovering all elements of $\boldsymbol{\Theta}$ (and $\boldsymbol{\Sigma}$ ) with $j$ multiple regression models. In relation to (1), the regression coefficients also have a direct mapping to partial correlation, that is

$$
\beta_{i j}=\rho_{i j \cdot z} \sqrt{\theta_{i i} / \theta_{j j}}
$$

This relationship is often utilized in the GGM literature. For example, with the regression coefficients in hand, it is possible to compute variance explained $\left(R^{2}\right)$ for each node in the network (Haslbeck \& Fried, 2017; Haslbeck \& Waldorp, 2018). This is typically done after the network structure has been determined, such that some elements of $\boldsymbol{\Theta}$ are zero. Hence, $R^{2}$ leads to a powerful inference in relation to the conditional relations for that node. Furthermore, there are a variety of approaches that use multiple regression to estimate $\boldsymbol{\Theta}$ (Liu \& Wang, 2017; Yuan, 2010) or that focus on the partial correlation matrix (Krämer, Schäfer, \& Boulesteix, 2009).

\section{Overfitting Revisited}

At this point, it is important to define overfitting: a model is said to be overfit when it is overly complex, such that it has learned noise in the (training) data and its predictions generalize poorly to unseen (test) data. Notice that there is nothing directly related to having a model that includes spurious associations or parameter estimation. The explicit focus is out-of-sample prediction.

In psychological networks, there are many nodes and this has raised concerns about overfitting. For example, with $p=20$ nodes there are 190 partial correlations in total. Although this sounds like an impressive number of parameters, the relation to multiple regression indicates that 19 regression coefficients are estimated $(p-1)$. Because the relation in (2) is exact, the dimensions of $\mathbf{Y}(n \times p)$ is of primary interest 
when thinking about estimation accuracy in GGMs. This is known as the "small $n$ large $p$ " problem:

When the matrix dimension $p$ is larger than the number of observations available $n$, the sample covariance matrix is not even invertible. When the ratio $p / n$ is less than one but not negligible, the sample covariance matrix is invertible, but numerically ill-conditioned, which means that inverting it amplifies estimation error dramatically. For large $p$, it is difficult to find enough observations to make $p / n$ negligible (p. 366, Ledoit \& Wolf, 2004).

Hence, there is an important distinction between the matrix dimensions and the number of parameters (see also p. 2 in Kuismin \& Sillanpää, 2017). With 190 partial correlations, and, say, $n=200$, this seems to warrant some form of regularization (" $p / n "=0.95$ ). In fact, it is common to motivate regularized estimation by noting the number of partial correlations (see for example p. 308 in Hevey, 2018). On the other hand, the $p / n$ ratio is 0.10 which does not seem problematic and it might be "negligible." These insights (and more) can be gleaned from decomposing the mean squared prediction error (MSE).

\section{Out-of-Sample Prediction}

First denote the training $\mathbf{Y}^{k}$ and test data $\mathbf{Y}^{-k}$, respectively. Both are distinct subsets of $\mathbf{Y}$. The training data is used to fit the initial regression model, say, predicting node $j$,

$$
\mathbf{Y}_{j}^{k}=\mathbf{Y}_{-j}^{k} \boldsymbol{\beta}_{j}^{k}+\boldsymbol{\varepsilon}^{k}, \boldsymbol{\varepsilon}^{k} \sim \mathcal{N}\left(\mathbf{0}, \sigma_{j}^{2} \mathbf{I}_{n}\right)
$$

where $\beta_{j}^{k}$ is a $(p-1) \times 1$ vector of regression coefficients, $\varepsilon$ is an $n$-dimensional vector with mean zero and covariance matrix $\sigma^{2} \mathbf{I}_{n}$. Note that $\sigma_{j}^{2}$ is the error variance for the $j$ th regression model ( $k$ is removed to simplify the notation), that is, $\Theta_{j j}^{k}=1 / \sigma_{j}^{2}$ in a GGM. This relation is critical for understanding overfitting in partial correlation networks, as it defines the absolute lower bound of MSE for the jth regression model - the irreducible error. With the predicted values for the test data defined as

$$
\hat{\mathbf{Y}}_{j}^{-k}=\mathbf{Y}_{-j}^{-k} \boldsymbol{\beta}_{j}^{k}
$$

the test error will typically be larger than $\sigma_{j}^{2}$. Although $\sigma_{j}^{2}$ is unknown, by drawing many random subsets and taking the average, the lower bound for MSE approximately corresponds to the residual variance from regressing $\mathbf{Y}_{j}$ onto $\mathbf{Y}_{-j}$. That is, nodewise predictability has an upper bound for accuracy that is defined by the diagonal elements of $\boldsymbol{\Theta}$. This allows for monitoring the reducible portion of MSE, that is indicative of overfitting, as $n$ approaches sample sizes representative of the network literature (Table 1 and 2 in Wysocki \& Rhemtulla, 2019).

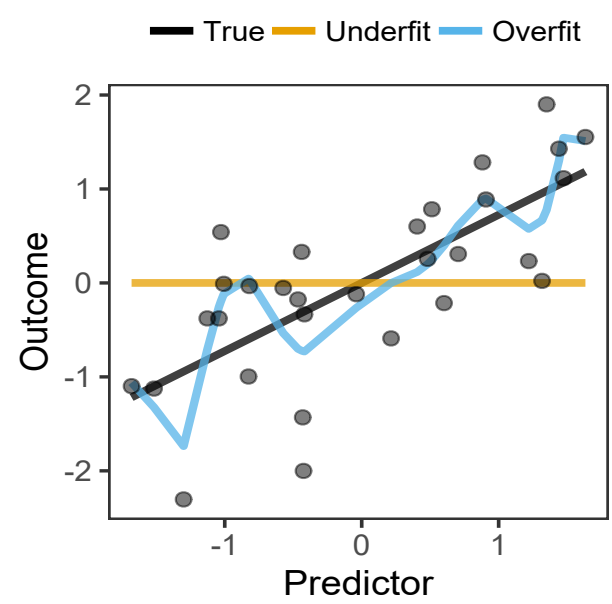

Figure 1. Regression models fitted in the motivating example, including the true model, an underfit model (intercept only), and an overift model (10th degree polynomial). The polynomial example is often used to highlight the dangers of overfitting, but it can also lead to an exaggerated concern if the context is not fully appreciated: typically the number of observations $(n)$ are fixed and model complexity is increased by adding polynomial terms $(p)$. However, psychometric scales are commonly used in network analysis and they have a specific number of items. This suggests that it is more reasonable to fix $p$ and then increase $n$ to representative sample sizes. Overfitting from these distinct scenarios is juxtaposed in Figure 2 (panel A and B).

\section{Theil MSE Decomposition}

We follow the econometric literature and now define the out-of-sample predictions, $\hat{\mathbf{Y}}_{j}^{-k}$, as $\mathbf{P}_{j}$ and the actual values for the test data, $\mathbf{Y}_{j}^{-k}$, as $\mathbf{A}_{j}$. MSE is the average squared distance, that is,

$$
\begin{aligned}
\mathrm{MSE} & =\mathrm{E}\left[\left(\mathbf{A}_{j}-\mathbf{P}_{j}\right)^{2}\right] \\
& =(\underbrace{\mathrm{E}\left[\mathbf{A}_{j}\right]-\mathrm{E}\left[\mathbf{P}_{j}\right]}_{\text {bias }})^{2} \\
& +\underbrace{\operatorname{Var}\left(\mathbf{A}_{j}\right)+\operatorname{Var}\left(\mathbf{P}_{j}\right)-2 \operatorname{Cov}\left(\mathbf{A}_{j}, \mathbf{P}_{j}\right)}_{\text {variance }} .
\end{aligned}
$$

Note that the variance term is specifically for the case of dependent variables, which accommodates the correlation between $\mathbf{A}_{j}$ and $\mathbf{P}_{j}$. The expression in (6) is commonly used outside of psychology (Murphy, 1988; Solazzo, Hogrefe, Colette, Garcia-Vivanco, \& Galmarini, 2017). Building upon this formulation, the lower bound for prediction error can be seen in the Theil decomposition for the sample-based MSE (Bento, Salvação, \& Guedes Soares, 2018; Fitzgerald \& Akintoye, 1995; Goodwin, 1997; Theil, 1961). This can be written as 


$$
\operatorname{MSE}\left(\mathbf{A}_{j}, \mathbf{P}_{j}\right)=\underbrace{\left(\overline{\mathbf{P}}_{j}-\overline{\mathbf{A}}_{j}\right)^{2}}_{\text {bias }}+\underbrace{\left(S_{\mathbf{P}_{j}}-r S_{\mathbf{A}_{j}}\right)^{2}}_{\text {var }}+\underbrace{\left(1-r^{2}\right) S_{\mathbf{A}_{j}}^{2}}_{m \cdot M S E} .
$$

Here the first term is the bias, and is simply defined as the squared difference between the averages for $\mathbf{P}_{j}$ and $\mathbf{A}_{j}$. This is much different than MSE for an estimator, as, in this case, the question of interest is the predicted and actual means for the test data. $S_{\mathbf{P}_{j}}$ and $S_{\mathbf{A}_{j}}$ denote the standard deviations for the predicted and actual values, respectively. The second term is the variance (Solazzo \& Galmarini, 2016). Note that this formulation arises from regressing $\mathbf{A}_{j}$ onto $\mathbf{P}_{j}$ (pp. 1 - 11, Miner \& Mincer, 1969), where $r^{2}$ is the coefficient of determination $\left(R^{2}\right)$ and the third term, "minimum achievable MSE," is the residual error. The advantage of (7) is that the first two terms will vanish with unbiased and efficient predictions (p. 65, Clements \& Hendry, 1998). Hence, the m.MSE component corresponds to the irreducible error (the residual variance in Equation 2). It is then useful to define U statistics in order to examine the contribution of each component to MSE (p. 139 Watson \& Teelucksignh, 2008). They are defined as

$$
\begin{aligned}
1 & =\mathrm{U}_{b}+\mathrm{U}_{v}+\mathrm{U}_{m} \\
& =\frac{\left(\overline{\mathbf{P}}_{j}-\overline{\mathbf{A}}_{j}\right)^{2}}{\mathrm{MSE}}+\frac{\left(S_{\mathbf{P}_{j}}-r S_{\mathbf{A}_{j}}\right)^{2}}{\mathrm{MSE}}+\frac{\left(1-r^{2}\right) S_{\mathbf{A}_{j}}^{2}}{\mathrm{MSE}} .
\end{aligned}
$$

Because they sum to one, each reflects a proportion of MSE. Furthermore, when $\mathrm{U}_{b}$ and $\mathrm{U}_{v}$ approach zero, $\mathrm{U}_{m}$ goes to unity such that predictive accuracy has reached its ceiling (i.e., MSE has been minimized, Clements \& Hendry, 1998). These properties are ideal for delving into the bias-variance tradeoff and overfitting. For example, $\mathrm{U}_{m}$ can be used to infer how close our model is to minimizing MSE, and thus, whether concerns of overfitting have been sufficiently quelled.

\section{Motivating Example}

In this section, we present an example of the bias-variance tradeoff. Recall that the bias-variance tradeoff is often used to discuss the benefits of regularization, and one contribution of this work is to provide an alternative perspective. Typically, demonstrative examples fix the number of observations and then increase the number of predictors in a regression model. We instead fix the number of variables $(p)$ and increase the number of observations $(n)$. This reflects psychological networks in particular, because nodes are typically items from psychometric scales that have a fixed number and $n$ is almost always much larger than $p$. This allows for monitoring $\mathrm{U}_{m}$ (minimum MSE) as $n$ gradually increases in size.
We also take this opportunity to delve into additional aspects of the bias-variance tradeoff and overfitting. For example, Gronau and Wagenmakers (2018) discussed "overfitting" in the context of a model with two parameters, that, in a predictive context, would be very unlikely to occur. By overfitting they meant the selected model included false positives. But as we show below, including too many variables in a model is not necessarily related to prediction error. Together, in addition to evaluating MSE in dimensions $(n \times p)$ representative of the network literature, we also tackle the relation between a model including too many variables and overfitting.

Because of the direct correspondence between multiple regression and the precision matrix (Equation 2), we followed the customary approach of using non-orthogonal polynomial terms to discuss the bias-variance tradeoff. This translates into a somewhat unrealistic design matrix that exhibits extreme multicollinearity between predictors (e.g., nearly infinite variance inflation factors). The true model is presented in Figure 1. The underlying data generating process is a linear relationship with $\beta_{0}=-5$ and $\beta_{1}=2$. In these experiments, the training data $\left(N_{\text {train }}\right)$ ranged from 11 to 250 (increments of 10), the test data size was fixed to $50\left(N_{\text {test }}\right)$, the SNR varied $(0.1 \text { and } 0.5)^{1}$, and the predictors were scaled to have mean zero and unit variance. All results were averaged across 1,000 trials for each condition.

Results. Figure 2 (panel A) shows the "dangers" of overfitting when $N_{\text {train }}$ is fixed to 50 and model complexity increases by including additional polynomial terms $(p / n \rightarrow$ $1)$. As a point of reference, the results are relative to the irreducible error (the residual variance) and on the logarithmic scale. Accordingly, the test (out-of-sample) error is minimized when it is closest to zero. The test error was minimized with the linear model that was used to generate the data (Figure 1, panel A). The test error then increased with model complexity, such that each additional predictor deteriorated prediction quality. On the other hand, the training (in-sample) error reduced with increasing model complexity. This is a classic case of overfitting: the model has "learned" noise in the training data and this then hinders generalizability to unseen data, say, that will be collected at some point in the future.

Figure 2 (panel C) includes an alternative perspective. In this case, the fitted model is always a $10^{\text {th }}$ degree polynomial ( $p$ is fixed) and $N_{\text {train }}$ gradually increases in size $(p / n \rightarrow 0)$. Recall that the true model is the linear relation in Figure 1 (panel A), and as such, the fitted model includes nine irrelevant variables that exhibit problematic multicollinearity. Here a much different pattern emerged than in panel B: both the training and test error converged to the irreducible error. It is striking how rapidly this occurred. Although the test error was (very) large with less than 100 observations,

\footnotetext{
${ }^{1} R^{2} \approx\{0.09,0.33\}$
} 


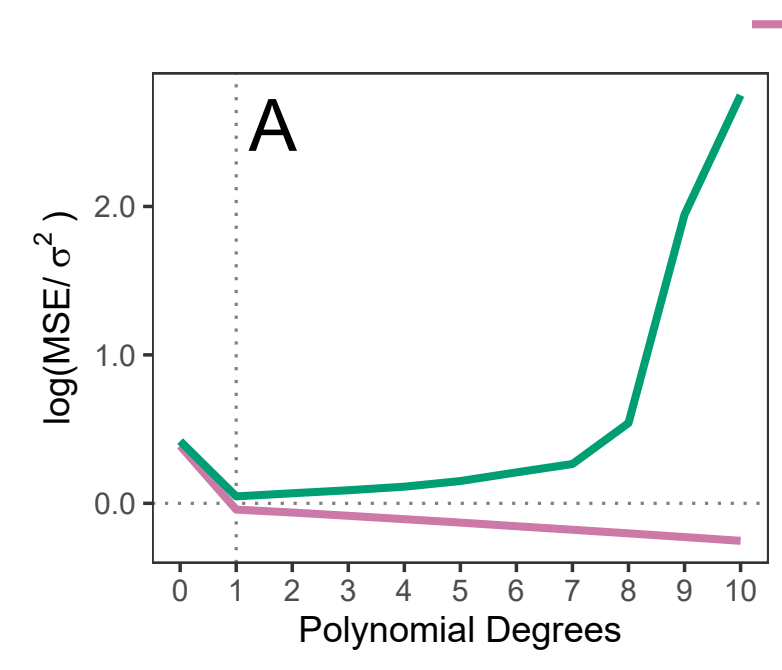

$-\mathrm{U}_{m}(\mathrm{~m} \cdot M S E)-\mathrm{U}_{b}($ bias $)-\mathrm{U}_{\mathrm{v}}(\mathrm{var})$

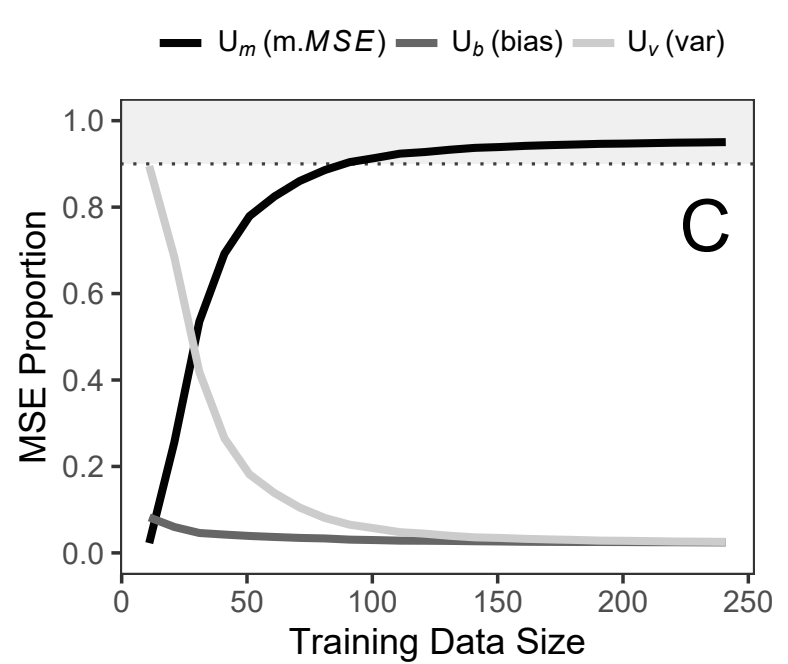

- Training - Test
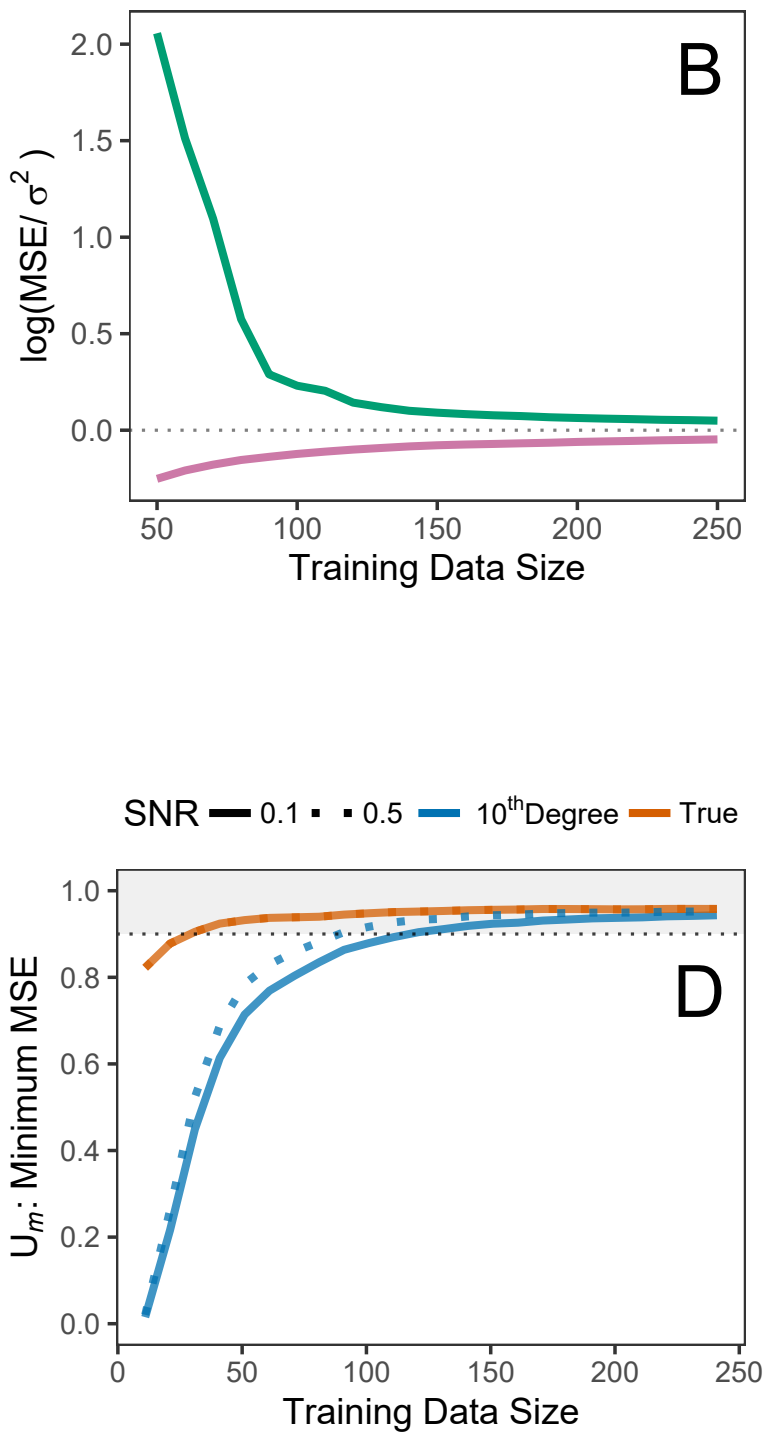

Figure 2. Results from the motivating example. Panel A includes a customary example of overfitting: the number of observations is fixed and model complexity increases. The $y$-axis denotes the ratio of mean squared error (MSE) to irreducible error (i.e., $\sigma^{2}$ in Equation 2). This is presented on the logarithmic scale, such that MSE is minimized at zero. As more terms are added to the model, training error becomes smaller than the irreducible error and test error dramatically increases. This is a classic case of overfitting. Panel B provides an alternative perspective: the number of variables is fixed (10th degree polynomial) and the number of observations increases. Here, even when not fitting the true model and dealing with extreme multicollinearity, test error is nearly minimized with 250 observations. Panel C decomposes of the test error in Panel B with Equation (7). Note that $\mathrm{U}_{m}$ (minimum MSE) is equal to one when the test error is minimized. This reveals that variance $\left(\mathrm{U}_{v}\right)$ contributes most to test error when the $p / n$ ratio is large, but this quickly dissipates and $\mathrm{U}_{m}$ rapidly approaches one. Panel $\mathrm{D}$ includes $\mathrm{U}_{m}$ for the 10th degree compared to the true model (Figure 1). This reveals that the former provides comparable predictions to the latter (SNR: signal-to-noise ratio). Together, overfitting is not omnipresent when the sample size approaches those representative of the network literature. This does not require fitting the true or even a realistic model. 
it approached the lower bound (i.e., the irreducible error) with only 150 observations. This is especially relevant for network analysis, where there is typically tens of nodes and hundreds of observations (Wysocki \& Rhemtulla, 2019).

We then decomposed the test error in Figure 2 (panel C). The idea here is to gain insight into the bias-variance tradeoff as $p / n \rightarrow 0$. These results are presented in panel D. As a point of reference, we subjectively defined 0.90 as an acceptable level of overfitting. That is, at least $90 \%$ of the systematic error due to bias and variance has been minimized. This can be inferred from monitoring $\mathrm{U}_{m}$ (minimum MSE), which is unity when the reducible portion of MSE has been completely minimized. In this example, $\mathrm{U}_{m}$ exceeds 0.90 with a sample size of around 100. Furthermore, with relatively few observations, it is clear that variance $\left(\mathrm{U}_{v}\right)$ dominates the test error. But this also reduces rapidly as $N_{\text {train }}$ gradually increases in size. It is also informative that the bias portion $\left(\mathrm{U}_{b}\right)$ is not a key contributor to MSE. Hence, it does not appear all that difficult to predict the mean of the test data.

Because our fixed $N_{\text {train }}$ experiments used a model that included too many variables, an interesting next step is then to compare performance to the true model. To this end, we again monitored $\mathrm{U}_{m}$ as the training data increased in size $(p / n \rightarrow 0)$. These results are presented in Figure 1 (panel E) and they are fascinating to behold: even when we fit the true model, prediction error was nearly identical to a $10^{\text {th }}$ degree polynomial when $N_{\text {train }}$ exceeded 150 . This was somewhat influenced by $R^{2}$, in that, with relatively more signal $(0.33$ vs. 0.09), the error more quickly approached that of the true model.

\section{Summary}

Together, these results demonstrate that overfitting does not seem to be all that bothersome. A similar finding was also observed in Brusco, Voorhees, Calantone, Brady, and Steinley (2019), where, even with a 10th degree polynomial ( $p=20$ and $n=900$ ), there was superior classification accuracy than more sophisticated approaches (e.g., support vector machine). In our example, with merely 250 observations, this was enough to mitigate extreme multicollinearity, practically minimize the reducible portion of MSE, and provide competitive performance compared to the true model. All the while we did not even select a model, which is often thought necessary to avoid overfitting. It is not. Of course, in network analysis the first objective is to estimate the underlying structure of conditional relations. Furthermore, perhaps overfitting can still be improved by employing $\ell_{1}$-regularization. These remaining questions are addressed in the next section.

\section{Significance Testing with Re-Estimation}

We propose nonregularized methodology specifically developed to fulfill the primary motivations for adopting $\ell_{1}$ regularization. First, our method not only limits spurious associations, but it preserves the relationship between specificity and the false positive rate (Williams \& Rast, 2019). Second, overfitting is mitigated by estimating a constrained precision matrix, given the estimated structure of conditional relations. The importance of these methodological desiderata are reflected in the applied network literature - the following brings them to fruition.

The first step follows Drton and Perlman (2004) and Williams and Rast (2019), both of which employed the Fisher- $z$ transformation for the sample partial correlations $r_{i j \cdot z}$. This transformation can be written as

$$
F\left(r_{i j \cdot z}\right)=0.5\left[\log \left(\frac{1+r_{i j \cdot z}}{1-r_{i j \cdot z}}\right)\right] .
$$

The resulting sampling distribution is (approximately) normal, which is one key advantage of employing (9). With $F\left(r_{i j \cdot z}\right)$ in hand, all that is needed to test

$$
\begin{aligned}
& \mathcal{H}_{0}: \rho_{i j \cdot z}=0 \\
& \mathcal{H}_{1}: \rho_{i j \cdot z} \neq 0
\end{aligned}
$$

is the sample size $(n)$ and the number of nodes $(p)$. With the number of nodes conditioned on denoted $c=p-2$ (i.e., those included in $z$ ), the standard error of $F\left(r_{i j}\right)$ is defined as $\frac{1}{\sqrt{n-3-c}}$. The edge set, $E$, is then constructed by rejecting $\mathcal{H}_{0}$ at a given $\alpha$ level. That is, for nodes $i$ and $j,(i, j) \in E$ if the test statistic, $(n-c-3)^{1 / 2}\left|F\left(r_{i j z}\right)\right|$, is larger than $\Phi^{-1}(1-\alpha / 2)$. Note that $\Phi$ denotes the cumulative density function of a standard normal distribution.

This straightforward approach has the advantage of being calibrated to the desired level of specificity $(1-\alpha$, Williams \& Rast, 2019). Although this can be used to limit spurious associations, thus fulfilling the first desideratum, there are issues for computing predictability. There are two options for obtaining nodewise predictions: (1) use the full model; or (2) use only the selected edges. Both would be employed after transforming the partial correlations to the corresponding regression coefficients with (3). However, the former does not reflect the estimated conditional dependence structure and the latter still uses estimates from the full model. Both options are less than ideal for computing predictability in GGMs.

To overcome these issues, we draw upon the wealth of methods from the GGM literature. These approaches are typically developed for high-dimensional data $(n<p)$. In this case, however, we borrow certain ingredients to improve the significance testing based recipe described in Williams and Rast (2019). We compute the maximum likelihood estimate for $\boldsymbol{\Theta}$, given the edge set, $E$, that was determined by rejecting $\mathcal{H}_{0}$ in (10). Let $\mathbf{A}$ denote the corresponding $p \times p$ adjacency matrix, that is, 


$$
\mathbf{A}_{i j}= \begin{cases}1 & \text { if } \rho_{i j} \neq 0 \\ 0 & \text { otherwise }\end{cases}
$$

where $\operatorname{diag}(A)=0$. The position of 1's in a given row corresponds to the neighborhood, $n e_{j}$, of conditional relations for node $j$ (i.e., the partial correlations determined to be statistically significant). We then follow the approach described in Hastie, Tibshirani, and Friedman (p. 634, 2008). This allows for estimating $\boldsymbol{\Theta}$ with a known structure of conditional relations. We refer to this approach as the "HTF" algorithm. Our innovation is to determine $\mathbf{A}$, which stands in for the known structure, with the hypothesis testing strategy in (10). To our knowledge, merging the HTF algorithm with hypothesis testing is a novel contribution to the GGM literature. We refer interested readers to (Chaudhuri, Drton, \& Richardson, 2007), where a similar approach was used to estimate a covariance matrix.

The basic idea is to maximize the likelihood, that is,

$$
\ell(\boldsymbol{\Theta})=\log (|\boldsymbol{\Theta}|)-\operatorname{trace}(\mathbf{S} \boldsymbol{\Theta})
$$

but with respect to constraints, or a pattern of zeros, that are defined by the adjacency matrix in (11). Note that $|\boldsymbol{\Theta}|$ denotes the determinant and $\mathbf{S}$ is the sample covariance matrix $(n-1)^{-1} \mathbf{Y}^{\prime} \mathbf{Y}$. Maximizing (12) is accomplished by further exploiting the relationship between $\boldsymbol{\Theta}$ and multiple regression (Equation 2). ${ }^{2}$ In this case, instead of working with the data matrix, $\mathbf{Y}, p$ subset regression models are fitted directly from the elements of $\mathbf{S}$. The subset of predictors (i.e., the neighbors of node $\mathbf{Y}_{j}$ ) are defined by $n e_{j}$ in (11). This gives "the correct solution and solves the constrained maximumlikelihood problem exactly" (p. 632, Hastie et al., 2008). Further details can be found in Emmert-Streib, Tripathi, and Dehmer (2019). Our particular implementation is summarized in the Appendix (Algorithm 1).

There are some important aspects of our approach that are worth emphasizing. First, we are not determining conditional relations via automated selection of $p$ multiple regression models. Rather, we are using significance testing directly on the partial correlations. This is not only computationally efficient, but it also avoids the known problems of making inference after performing model selection (e.g., Hurvich \& Tsai, 1990; Leeb \& Pötscher, 2005). Second, if predictability is of interest, estimating $\boldsymbol{\Theta}$ with some elements constrained to zero provides regression coefficients that reflect the conditional dependence structure. Merging this into a hypothesis testing framework should allow for enjoying the predictive properties of nonregularized estimation (e.g., Figure 1 in Williams et al., 2019), all the while having a defined error rate. This fulfills both desiderata. Third, there is one caveat of estimating a precision matrix with a given conditional dependence structure: it can be used for prediction but it would be incorrect to make inference (e.g., computing $p$ - values) from the estimates. This would be akin to computing, say, confidence intervals (CI) for a subset of variables that were selected in multiple regression. This is known to result in model selection bias, given that both $p$-values and CIs assume the model was fixed in advance (it was not selected from the data). For an overview of this topic, we refer interested readers to Taylor and Tibshirani (2015) and Berk, Brown, and Zhao (2010).

\section{Proposed Method}

To summarize, our method is implemented with the following steps:

1. Compute the Fisher-z transformations of the $p(p-1) / 2$ partial correlation coefficients and test their significance at a desired $\alpha$ level.

2. Construct a symmetric $p \times p$ adjacency matrix, $\mathbf{A}$, with $a_{i j}=a_{j i}=1$ if the partial correlation between nodes $i$ and $j$ was statistically significant and $a_{i j}=a_{j i}=0$ otherwise.

3. Apply the HFT algorithm in Appendix 1 to estimate $\boldsymbol{\Theta}$, given $\mathbf{A}$ and the sample covariance matrix $\mathbf{S}$.

\section{Simulation Studies}

This section includes three simulation studies. In each, real datasets are used from the network literature. The key difference between the experiments is the measure for test error. The first two assess prediction error from multiple regression (large holdout set and leave-one-out crossvalidation), as when computing predictability, and the third investigates test error for the entire precision matrix. Recall that our method (herein referred to as Nonreg $_{N H S T}$ ) provides an estimate of $\boldsymbol{\Theta}$, given the estimated graph (Equation 11), and hence experiment three provides key insights into the accuracy of our proposal.

For comparison, we included the default methodology in psychology that employs $\ell_{1}$-regularization in combination with the extended Bayesian information criterion (EBIC) for selecting the tuning parameter (herein referred to as $\left.\mathrm{Glasso}_{E B I C}\right)$. As a point of reference, we also included the sample covariance matrix (a full model). For our method, we set $\alpha=0.10$ that corresponds to $90 \%$ specificity (see Figure 1 in Williams \& Rast, 2019). Although this choice is arbitrary, it parallels our subjectively defined level of acceptable overfitting (i.e., $90 \%$ of the irreducible error). For Glasso ${ }_{E B I C}$, we used the default settings in the R package qgraph (Epskamp, Cramer, Waldorp, Schmittmann, \& Borsboom, 2012).

\footnotetext{
${ }^{2}$ We explored the possibility of directly maximizing (12) with convex optimization, but it was much slower than the HTF algorithm.
} 


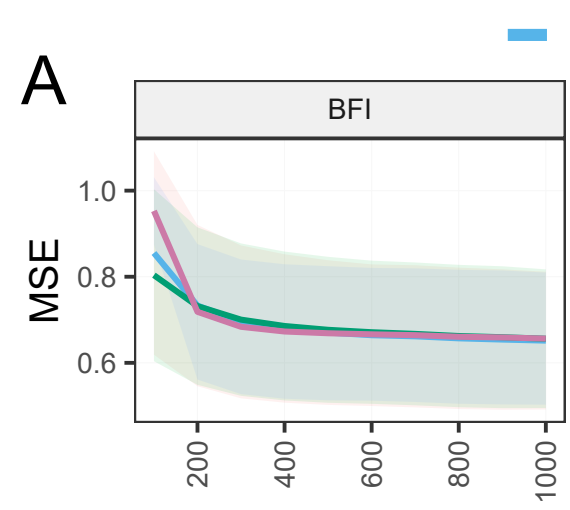

Full $=$ Nonreg $_{N H S T}=$ Glasso $_{E B I C}$
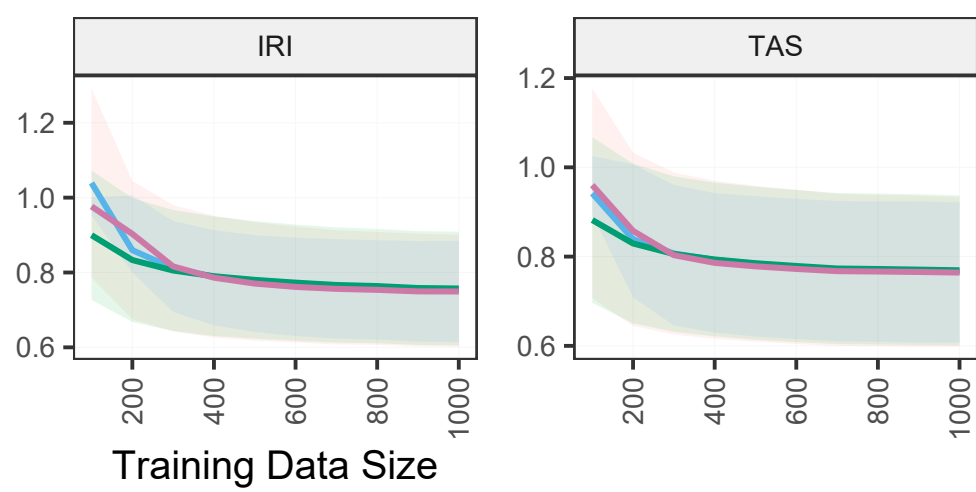

B
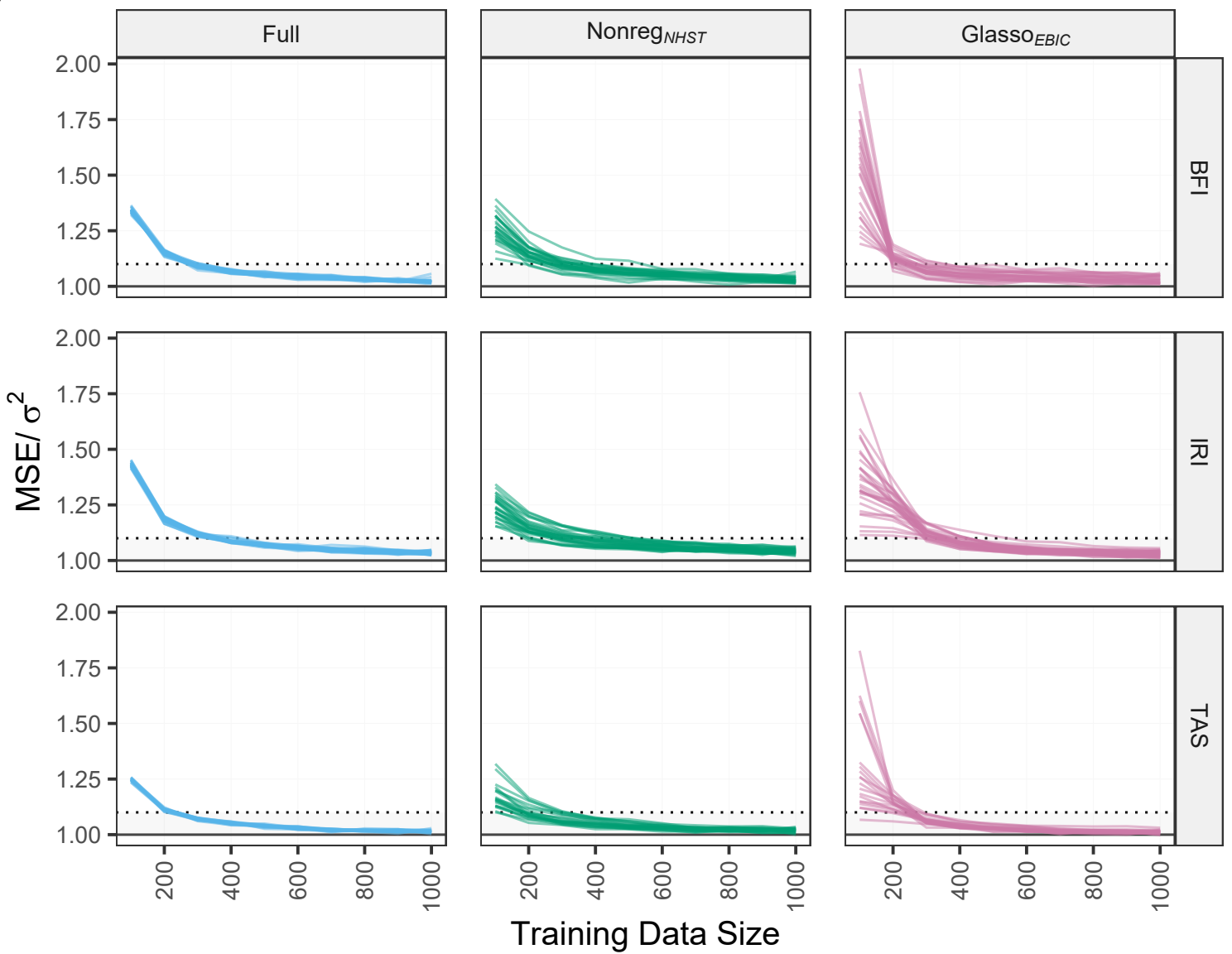

Figure 3. Simulation results for Experiment 1. In panel A, test error was averaged across each node in the respective networks. Each line denotes a network estimation method, the $y$-axis is MSE, and the ribbons denote $\pm 1 \mathrm{SD}$. Across almost all training sizes there were not any notable differences between methods in predictive accuracy. The one exception being low sample sizes, wherein both nonregularized methods outperformed Glasso ${ }_{E B I C}$ (see text for explanation). In panel B, each line corresponds to test error for each node. These were averaged across in panel A. The dotted line corresponds to a $10 \%$ increase from the irreducible error. The solid line is the best possible predictive performance. The methods again provide similar predictive performance and the vast majority of test error is minimized. Together, there is not a readily discernible advantage of Glasso $_{E B I C}$, and even the full model appears to mitigate overfitting in sample sizes representative of the network literature (Table 1 in Wysocki \& Rhemtulla, 2019). BFI $(p=25)$, IRI $(p=28)$, and TAS $(p=20)$. 


\section{Illustrative Data}

Dataset 1. This dataset come from the R package psych (Revelle, 2019). There are 25 self-reported items that measure personality in 2236 subjects. The items were taken from the international personality item pool. There are five factors: agreeableness, conscientiousness, extraversion, neuroticism, and openness. The majority of subjects were between 20 and 60 years old $(\mathrm{M}=29.5$ years, $\mathrm{SD}=10.6$ years $) .32 \%$ were males and $68 \%$ were females. The items are scored from 1 to 6 , where " 1 " corresponds to "very inaccurate" and 6 corresponds to "very accurate."

Dataset 2. This dataset comes from a network study using the Interpersonal Reactivity Index (IRI) to measure empathy in 1973 subjects (Briganti, Kempenaers, Braun, Fried, \& Linkowski, 2018). Further details about the IRI can be found in Davis (1983). The subjects were between 17 and 25 years old $(\mathrm{M}=19.6$ years, $\mathrm{SD}=1.6$ years $) .57 \%$ were females and $43 \%$ were males. This scale includes 28 items meant to assess four components of empathy: fantasy, perspective taking, empathic concern, and personal distress. The items are scored from 0 to 4 , where " 0 " corresponds to "Doesn't describe me very well" and " 4 " corresponds to "Describes me very well."

Dataset 3. This dataset comes from a network study using the Toronto Alexithymia Scale (TAS) as assessment tool (Briganti \& Linkowski, 2019). Further details about the TAS can be found in Bagby, Parker, and Taylor (1994). There are 1925 subjects that range between 17 and 25 years old $(\mathrm{M}=$ 19 years; $\mathrm{SD}=1.6$ years). $58 \%$ of them were females and $42 \%$ were males. This scale includes 20 items that measure alexithymia in three domains: difficulty identifying feelings, difficulty describing feelings and externally-oriented thinking. The items are scored from 1 to 5, where " 1 " corresponds to "I completely disagree" and "5" corresponds to "I completely agree."

\section{Experiment 1}

In this experiment, we followed the approach described in (Leppä-aho, Pensar, Roos, \& Corander, 2017). We first randomly sampled non-overlapping training and test sets from the full datasets, and then estimated the network structure from the training data. After transforming the selected precision matrix into the corresponding regression coefficients (Equation 2), the test error (MSE) was computed for each node in the respective networks. The test data size was fixed to 50 (48 was used in Leppä-aho et al., 2017) and the training data ranged in size from 100 to 1000 (in increments of 100). This corresponds to maximum $p / n$ ratios of 0.28 (IRI), 0.25 (BFI), and 0.20 (TAS). These ratios gradually diminish with larger training sets (i.e., $p / n \rightarrow 0$ ). Note that we did want to include larger ratios, but with smaller $n$, Glasso ${ }_{E B I C}$ would select only empty networks (or intercept only models). The results were averaged across 1000 simulations trials.
Results. We first describe the results in Figure 3 (panel A), where the test error was averaged across nodes. The methods provided very similar performance (often within three decimal places). This can be inferred from the overlapping lines and large uncertainty (the ribbons correspond to \pm one SD). The one distinction between models emerged with the largest $p / n$ ratios. This was paradoxical, however, in that both the fully model and Nonreg ${ }_{N H S T}$ provided more accurate predictions than Glasso ${ }_{E B I C}$. This can be understood in reference to the motivating example, where, with no polynomial terms (i.e., an intercept only model), the test error was large due to underfitting (Figure 1, panel A). In this case, the networks selected with Glasso ${ }_{E B I C}$ included too few edges. We return to the tradeoff between sparsity (or parsimony) and predictive accuracy below (Section Illustrative Example).

These results also highlight a key point we emphasized above: the distinction between the dimensions of $\mathbf{Y}(n \times p)$ and the number of partial correlations. Recall that the latter is often emphasized when motivating $\ell_{1}$-regularization. In the IRI data, although there are 378 partial correlations, the test error for Nonreg $\operatorname{NHST}_{N H}$ reduced from $0.789\left(N_{\text {train }}=400\right)$ to $0.754\left(N_{\text {train }}=1000\right)$. Of course, a difference of 0.035 could be quite important, depending on the application, but it highlights that the most problematic situations are large $p / n$ ratios. This can be seen in the motivating example (Figure 2, panel $\mathrm{C}$ ), where the variance proportion $\left(\mathrm{U}_{v}\right)$ of test error was largest with $p / n \approx 0.91$. The test error was not much different for Glasso ${ }_{E B I C}(0.788$ and 0.748$)$. In fact, with $N_{\text {train }}=400$ and 378 partial correlations $(p=28)$, the difference between the full model and Glasso $E B I C$ was merely 0.004 !

Interestingly, as the training data increased in size $(p / n \rightarrow$ $0)$ the nonregularized methods did not have superior performance. Of course, our central message is that overfitting might not actually be all that problematic in psychological networks. In these results, it appears that Glasso ${ }_{E B I C}$ does not mitigate overfitting compared to nonregularized estimation. And note that, when $n$ increases, Glasso ${ }_{E B I C}$ is known to have an inflated false positives (Williams \& Rast, 2019; Williams et al., 2019).

We now describe the results in Figure 1 (panel B), where each line corresponds to the average test error for each node. Because we randomly sampled test and training sets, the irreducible error should be close to the residual variance from the full dataset. This was described above. The results are presented relative to the irreducible error. As a point of reference, the dotted line at 1.1 indicates the MSE is $10 \%$ larger than the irreducible error. These results reveal that, even with $N_{\text {train }}=400$, most nodes are below 1.1. In other words, the vast majority of reducible error has been minimized. This pattern held for each model and dataset. When comparing models, the results were very similar ${ }^{3}$. The most notable

\footnotetext{
${ }^{3}$ We have provided all the simulation results online for re-
} 
differences were again with the smallest sample sizes, where nonregularized approaches often had superior performance. On the other hand, with the larger samples, the performance of Glasso ${ }_{E B I C}$ improved to be comparable and sometimes better than the nonregularized methods.

Perhaps most striking was the performance of the full model. Recall that this corresponds to a fully connected network. The node specific estimates were far less variable than Nonreg $_{N H S T}$ and Glasso $E B I C$. This was likely due to model selection inducing variability in the estimates. Furthermore, at the level of the individual nodes, the full model regularly outperformed Glasso ${ }_{E B I C}$ (although, again, these differences were very minor). Together, overfitting hardly seems to be a "severe challenge" that warrants regularized estimation.

\section{Experiment 2}

In this experiment, we investigated test error with leaveone-out cross-validation (LOOCV). Rather than take a random set, as in experiment 1, MSE was computed from all observations in the dataset. One advantage of this approach is that it more accurately reflects how predictability would be assessed in applied settings. Because we are still interested in the $p / n$ ratio, we took subsets that ranged in size from 100 to 1000 (in increments of 100). For each subset, the network was first selected with row $k$ removed and then the observations from row $k$ were predicted. This was done after transforming the elements of the selected precision matrix to the corresponding regression coefficients (Equation 2). This was done for each row in the subset of data $\left(k=1, . ., N_{\text {subset }}\right)$, after which the test error was computed. Note that we sequentially moved through the rows, such that, with $N_{\text {subset }}=100$ and $N_{\text {subset }}=200$, the error was computed for rows 1 through 100 and 200, respectively. This was done to ensure the results were not susceptible to a single, random partition, of the data.

Results. We first discuss Figure 4 (panel A). The test error was averaged across nodes. The results were similar to experiment 1 , in that the methods ultimately converged to be similar to one another. However, there were some differences with the largest $p / n$ ratios. These again favored Nonreg $_{N H S T}$. This was most apparent for the IRI dataset $\left(N_{\text {subset }}=100\right.$ to 400), where the nonregularized predictions were more accurate than Glasso $E B I C$. Interestingly, the full model provided competitive performance for the vast majority of conditions. This was especially the case for sample sizes larger than 300 . We emphasize that this lends further credence to the notion that reducing the number of parameters is not necessary to mitigate overfitting.

Panel B includes a different perspective on the same results. Rather than averaging across nodes, or noting what were typically small differences in test error, we computed the proportion of nodes in which the test error was minimized by each method. The basic idea here is to gain insight into what might be observed if using both methods in an ap- plied settings. The top row includes our proposed method. Again, there was not a uniformly superior method. However, Nonreg $_{N H S T}$ minimized the error in more than half of the nodes in a given network 18 times, whereas, for Glasso ${ }_{E B I C}$, this occurred only 9 times in total. The largest disparity was for the BFI and IRI data. Our nonregularized method was favored in $70 \%(14 / 20)$ of the conditions (as indicated by minimizing the error in more than half of the nodes) and Glasso $_{E B I C}$ was favored in $25 \%(5 / 20)$. Note that there was one tie, where each method minimized the test error for half of the nodes (IRI dataset with $N_{\text {subset }}=100$ ).

The bottom row includes the results for the full model. Glasso $_{E B I C}$ had notably better performance. For example, in 18 compared to 11 conditions the regularized method minimized the test error in more than half of the nodes. It is important to note that regularization is used for network analysis because overfitting is thought to be a severe challenge. However, these results further reveal that, even with models including hundreds of parameters, the advantage for Glasso $_{E B I C}$ is not overwhelming. Indeed, if both the full model and Glasso ${ }_{E B I C}$ were both computed side-by-side for a given dataset, some nodes would be better predicted by the full model - and in some cases the majority of nodes in a network! This is striking, because it is just these situations

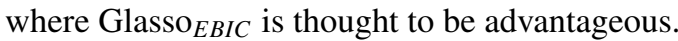

\section{Experiment 3}

In this experiment, we investigated cross-validation error for the entire precision matrix. This can be understood as global measure of predictive accuracy. This was accomplished by computing the log-likelihood for the selected precision matrix estimated from the training data and the covariance matrix estimated from the test data (Equation 12). Note that the likelihood is also known as the log predictive density (section 2 Gelman, Hwang, \& Vehtari, 2014) and is commonly used to select the tuning parameter in glasso (Bien \& Tibshirani, 2011; Gao, $\mathrm{Pu}, \mathrm{Wu}, \& \mathrm{Xu}, 2009)$. In this case, we are using it to compare methods in terms predictive accuracy. Furthermore, this experiment also provides key insights into the accuracy of using classical significance testing to determine the missing edges, that is, those set to zero in (11). For each of 1000 simulation trials, we randomly sampled nonoverlapping training and test sets from the full dataset. The test data size was fixed to 50 and the training data ranged in size from 100 to 1000 (increments of 100).

Results. Figure 5 includes these results. Higher scores indicate superior predictive performance (i.e., maximizing the likelihood). These results reveal that using nonregularized estimation in combination with null hypothesis significance testing provides an accurate estimate of the precision

searchers to explore and possibly find notable patterns that we overlooked. 


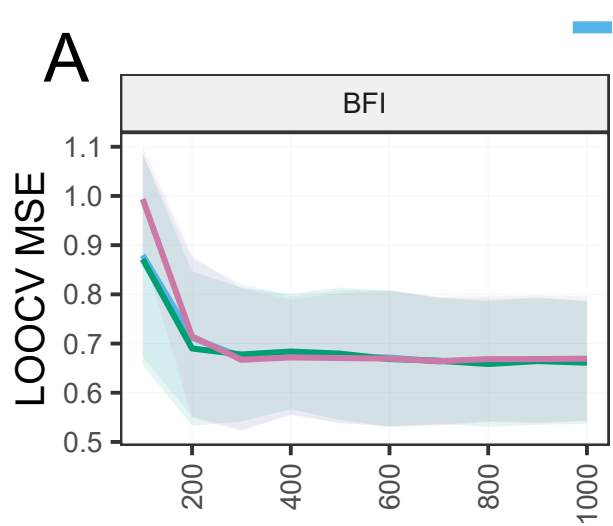

Full $=$ Nonreg $_{N H S T}=$ Glasso $_{E B / C}$
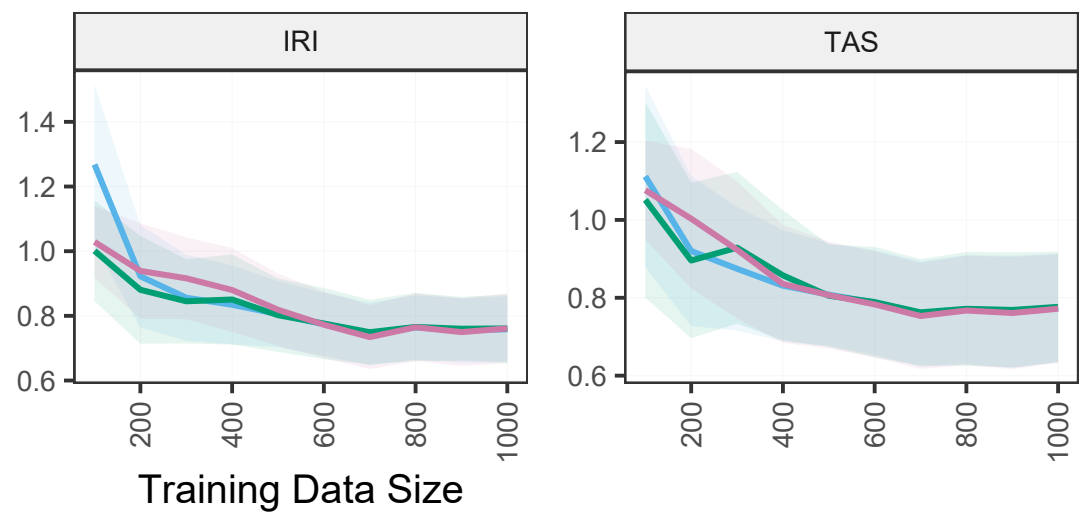

B

Full Nonreg $_{N H S T} \quad$ Glasso $_{E B I C}$
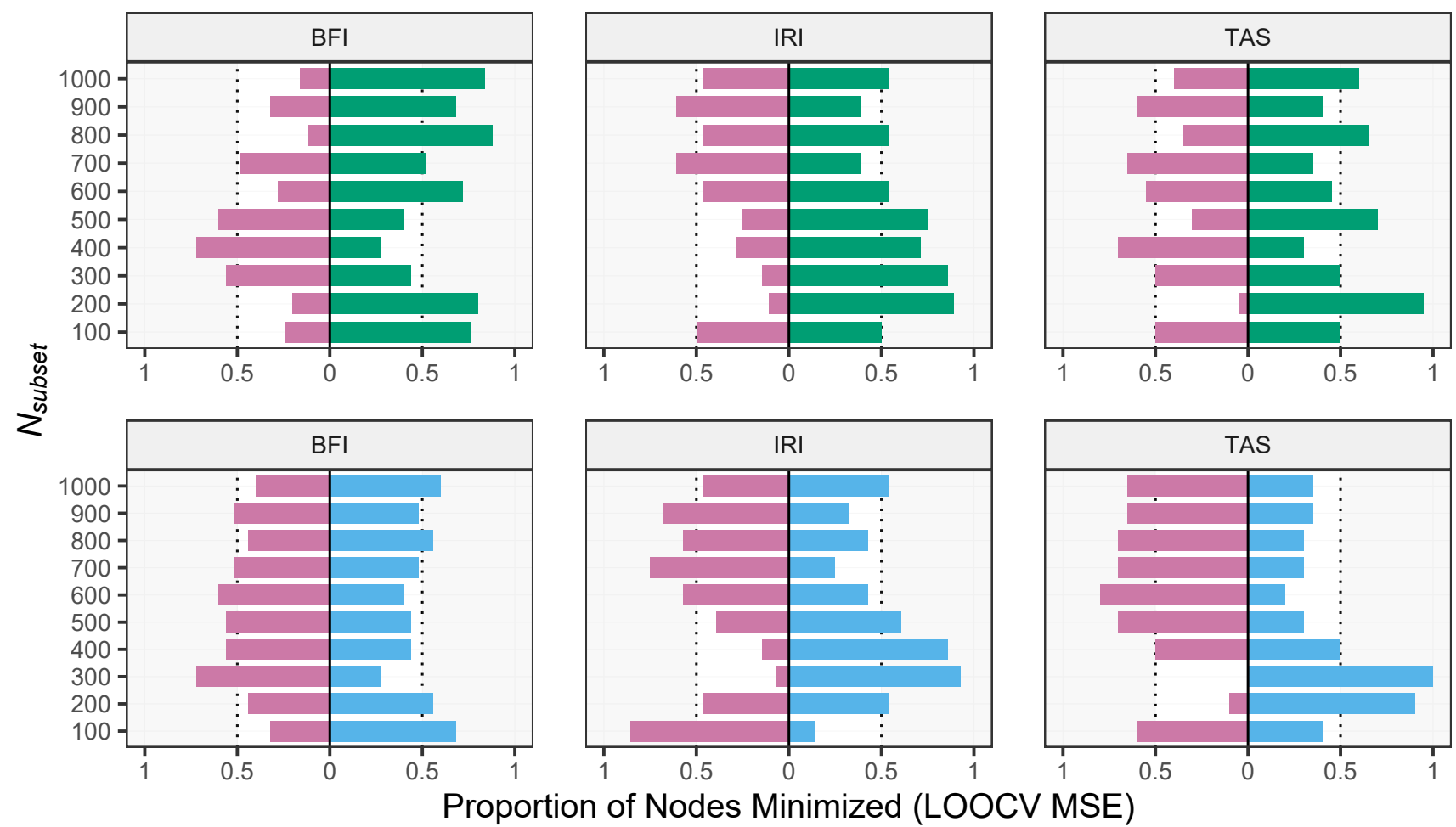

Figure 4. Simulation results for Experiment 2. Panel A includes leave-one-out cross-validation (LOOCV) error averaged across nodes in the respective networks. Each line denotes a network estimation method, the $y$-axis is LOOCV MSE, and the ribbons denote $\pm 1 \mathrm{SD}$. This reveals an advantage for the nonregularized methods with small training data sizes (see text for explanation). The methods quickly converged to have similar predictive accuracy. Panel B provides a different perspective on the same results. For each training size, we computed the proportion of nodes in which each method had the lowest test error. The grey region corresponds to $\geq 50 \%$ of the nodes. In the top row, Nonreg ${ }_{N H S T}$ and Glasso ${ }_{E B I C}$ minimized MSE in more than half of the nodes 18 and 9 times, respectively. In the bottom row, Glasso $E B I C$ and the full model minimized MSE in more than half of the nodes 18 and 11 times, respectively. This indicates an advantage of Glasso $E B I C$ compared to the full model, but not when compared to our method $\left(\operatorname{Nonreg}_{N H S T}\right)$. BFI $(p=25)$, IRI $(p=28)$, and TAS $(p=20)$. 

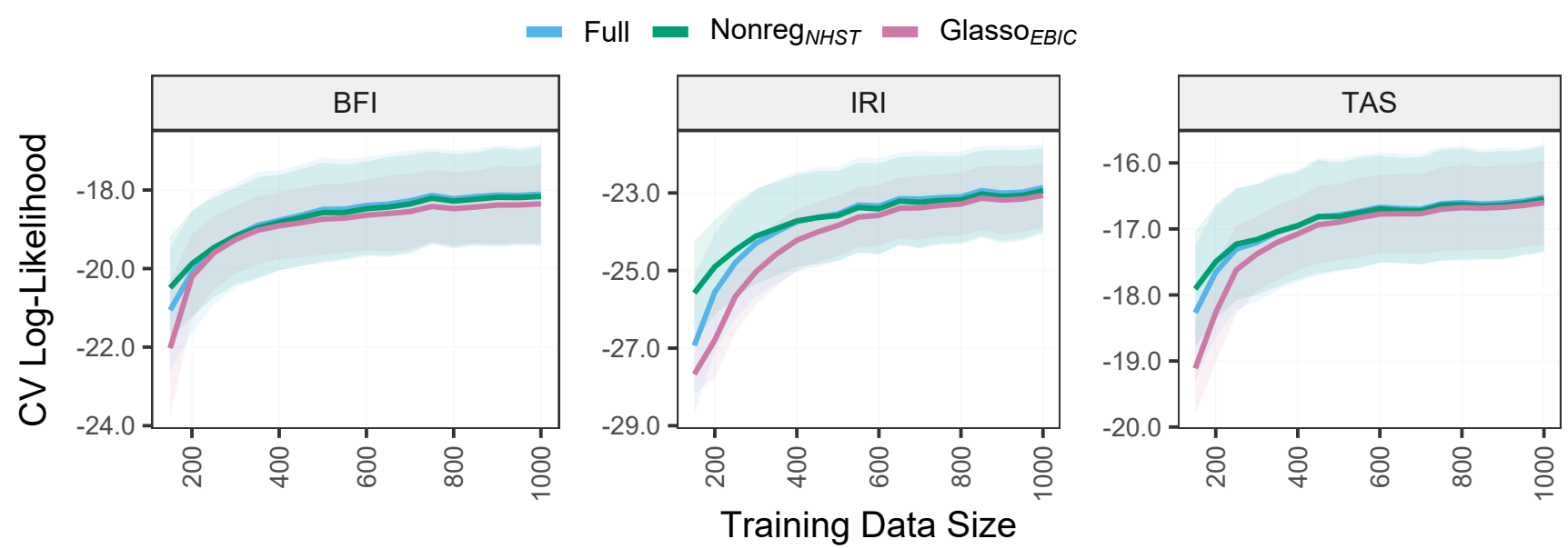

Figure 5. Simulation results for Experiment 3. The $y$-axis is the cross-validated log-likelihood (Equation 12). This provides a measure of global predictive accuracy for the network. Larger values indicate superior performance (i.e., maximizing the likelihood). The nonregularized methods often provided more accurate predictions than Glasso ${ }_{E B I C}$. This demonstrates: (1) the accuracy of our proposed method for estimating a constrained precision matrix (Algorithm 1); and (2) regularization is not needed to mitigate overfitting (e.g., even the full model provided more than competitive performance). BFI ( $p=25)$, IRI $(p=28)$, and TAS $(p=20)$.

matrix with zeros. In fact, Nonreg $_{N H S T}$ often had superior predictive performance compared to Glasso ${ }_{E B I C}$. Furthermore, it is also apparent that even the full model provided excellent performance compared to Glasso ${ }_{E B I C}$. There was also large uncertainty for each method, but it is still informative that the averages were better for the nonregularized methods. The benefit of constraining the precision matrix is also readily apparent. For example, while the differences were small, our proposed method was more accurate than the full model with the largest $p / n$ ratios. All three methods ended up converging to be quite similar as $p / n \rightarrow 0$. Together, when considering the entire GGM, the nonregularized method provided more than competitive predictions.

\section{Summary}

Together, these results do not provide support for the thought that regularization is needed to mitigate overfitting in psychological networks. Recall that one motivation for adopting $\ell_{1}$-regularization was the worry of overfitting (due to estimating a large number of partial correlations). However, these results show that, even with more partial correlations than observations, even the full model provides more than competitive performance. And, in turn, our proposed method provides both an estimate of the conditional dependence structure and accurate predictions. These findings can be understood in relation to the correspondence between multiple regression and the precision matrix (Equation 2). In the IRI dataset, there are 28 items resulting in 378 partial correlations. This might seem to be an especially difficult situation with, say, a sample size of 400 . However, with $p$ defined in reference to the dimensions of $\mathbf{Y}$ (i.e., the number of nodes), the $p / n$ ratio is merely 0.07 . This provides a fresh perspective into overfitting, or lack thereof, in psychological networks.

\section{Illustrative Example}

In this section, we discuss strategies for using our proposed method in applied settings. The most pressing question relates to correcting for multiple comparisons, as determining the edge set $E$ requires potentially hundreds of tests. Two approaches to address this issue would be to employ, say, a Bonferroni corrected alpha level or simultaneous confidence intervals (e.g., Drton \& Perlman, 2004). This would reduce spurious findings and provide a sparse network. However, the motivating example and simulation results point to a tradeoff between reducing spurious associations and predictive accuracy. For example, a stringent alpha level will necessarily reduce statistical power and potentially deteriorate predictive accuracy by detecting too few edges (i.e., underfitting). While using a data-driven approach for choosing $\alpha$ would violate the foundations of frequentist inference, it is still informative to assess the desired level of specificity ( $1-\alpha$, Williams \& Rast, 2019) in relation to prediction error. This can be used to guide the choice of a particular multiple testing strategy.

We simulated 1000 trials with non-overlapping, randomly sampled training and test data. We used training sets of size $N_{\text {train }}=250,500,1000$. The test data size was fixed at $N_{\text {test }}=50$. For each training size, the network structure was estimated using alpha levels of $\alpha=$ $0.05 / t, 0.01,0.05,0.1$, and 0.2 . $t$ is the respective number of tests conducted for each dataset (i.e., the number of partial 

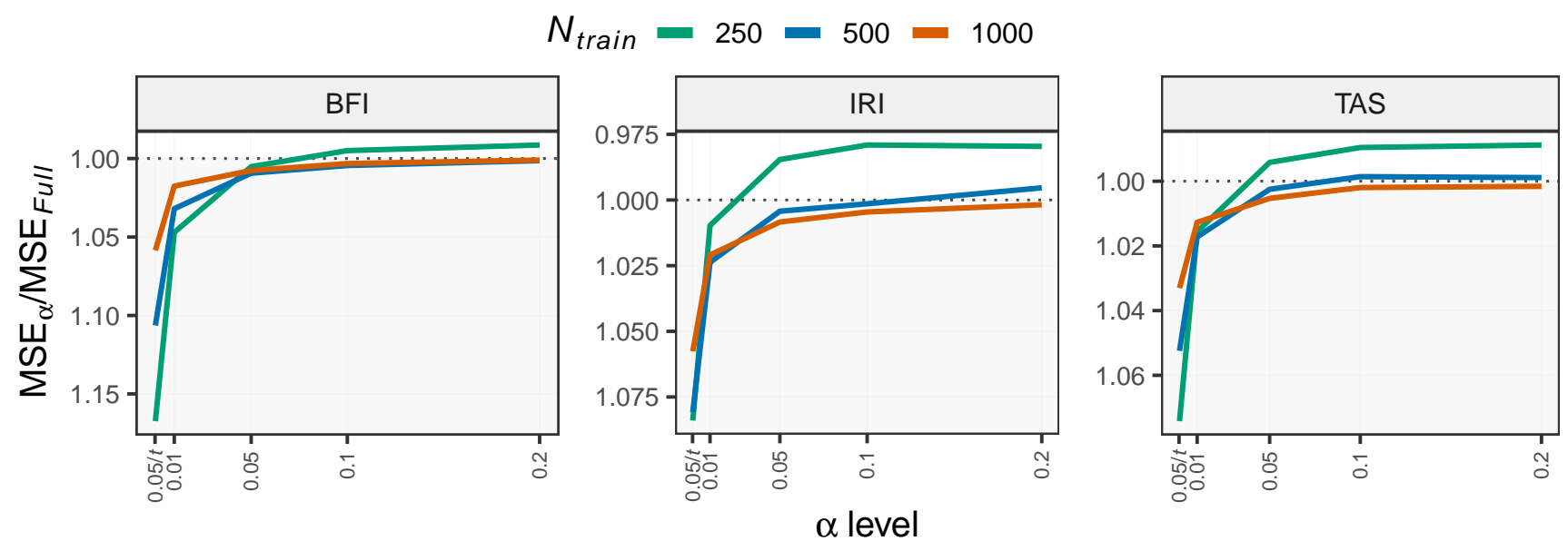

Figure 6. Simulations results for the Illustrative Example. Each color indicates the sample size used to train the model. The lines correspond to the test error for each $\alpha$ level $\left(\mathrm{MSE}_{\alpha}\right)$ relative to that of the full model $\left(\mathrm{MSE}_{F u l l}\right)$. Values less than one indicate inferior predictive performance compared to the full model (i.e., the grey region). This reveals: (1) predictive accuracy was best at higher alphas across all conditions, that is, a network that included more spurious associations minimized test error; and (2) the full model routinely provided the best performance and this was always the case for alpha levels less than 0.05 . This points towards a methodological quagmire: rigorous inferential standards can deteriorate predictive accuracy (due to reducing power and thus underfitting). BFI $(p=25)$, IRI $(p=28)$, and TAS $(p=20)$.

correlations tested), and $0.05 / t$ is a Bonferroni corrected alpha level.

\section{Results}

Figure 6 displays the results, where the test error (MSE) was computed relative to the full model. Hence, values less than one indicate superior predictive performance of the full model. A clear tradeoff emerged between alpha level and predictive accuracy. Across all conditions, predictive accuracy improved as alpha increased. However, this increase in prediction accuracy came at the cost of an inflated false positive rate - that is, correcting for multiple tests, or even just setting traditional alpha levels (i.e., 0.05) did not lead to the best predictions. Instead it was larger alpha levels that routinely minimized the test error. The tradeoff between a desired error rate and better predictive power is most evident in lower sample sizes. For example, the Bonferroni correction is overly stringent at $N_{\text {train }}=250$. This is evidenced by having the worst predictive accuracy among all alpha levels. On the other hand, predictive accuracy was maximized with more liberal alpha levels than traditionally used (e.g., 0.20 vs. 0.05 ). This points towards a predicament: rigorous inferential standards appear to come at the cost of inferior predictive accuracy.

\section{Discussion}

In this work, we delved into the important topic of overfitting and predictability in psychological networks. We set out to investigate whether overfitting is a "serious challenge" that requires $\ell_{1}$-regularization. We first presented a motivating example that was based upon a polynomial regression. Here important aspects of the bias-variance tradeoff were highlighted in low-dimensional data that are commonplace in the network literature $(p<n)$. This included a mean squared error (MSE) decomposition, where it was apparent that bias and especially variance are most problematic with $p / n$ ratios that are rarely encountered. Additionally, with merely 250 observations this was enough to overcome extreme multicollinearity and nearly minimize the reducible portion of MSE.

We then introduced novel methodology fulfilling two methodological desiderata: (1) reducing or controlling spurious associations and (2) mitigating concerns of overfitting by providing accurate predictions. These were the primary motivations for adopting $\ell_{1}$-regularization in the network literature. In extensive simulations, we demonstrated that nonregularized estimation provides more than competitive predictive accuracy. This complements recent work showcasing the inflated false positive rate inherent to $\ell_{1}$-regularization (Williams \& Rast, 2019; Williams et al., 2019). Together, it is nonregularized, as opposed to regularized estimation, that best satisfies these desiderata.

An additional contribution of this work clarified overfitting. We tackled this important topic because the word "overfitting" is used far too loosely in the social-behavioral sciences. For example, while Babyak (2004) argued that "overfitting" can result in failing to reproduce findings, prediction was never actually examined! In Babyak (2004), the term "overfitting" was used to reference a model that in- 
cludes spurious relations. Recall that overfitting does not actually refer to replicating, say, significant effects or spurious associations, but predicting unseen data. The residue of this confusion impedes methodological literacy and practice: when focusing on reducing spurious relations, this can actually deteriorate prediction quality in situations common to the network literature (Figure 6). This is well known in, say, the forecasting literature, where selecting the true model (or the most parsimonious) is not an omnipresent goal "even if there was a true underlying model, selecting that model will not necessarily give the best forecasts" (p. 165, Hyndman \& Athanasopoulos, 2018). This is due to inherent tension between model selection consistency ${ }^{4}$ and minimizing the mean squared error, that is, "for any model selection criterion to be consistent, it must behave suboptimally for estimating the regression function" (p. 937, Yang, 2005). The latter matters most for prediction, whereas consistent model selection limits spurious associations.

Furthermore, the worry of overfitting has ramifications that extend beyond prediction to inference. To date, $\ell_{1}$ regularization is used for both inference and prediction in the network literature. This is unfortunate. Regularized estimation does not readily allow for inference, and, in fact, obtaining valid $p$-values and confidence intervals is still an active area of research (see references in Zhang, Ren, \& Chen, 2018). Inherent to Glasso ${ }_{E B I C}$ is data-driven model selection and this presents further challenges for inference:

We do not discuss statistical inference...(e.g., looking at $p$-values associated with each predictor). If you do wish to look at the statistical significance of the predictors, beware that any procedure involving selecting predictors first will invalidate the assumptions behind the $p$-values. The [data-driven] procedures we recommend for selecting predictors are helpful when the model is used for [prediction]; they are not helpful if you wish to study the effect of any predictor (p.168, Hyndman \& Athanasopoulos, 2018).

This induces model selection bias (Danilov \& Magnus, 2004; Kabaila, 1995; Leeb \& Pöscher, 2006; Leeb, Pötscher, \& Ewald, 2015) and requires post-selection corrections (Berk, Brown, Buja, Zhang, \& Zhao, 2013; Lee, Sun, Sun, \& Taylor, 2016; Taylor \& Tibshirani, 2017). Because Glasso $E$ EIC employs a penalty and data-driven model selection, this results in an underappreciated double whammy: (1) penalization compromises the sampling distribution of the partial correlations by introducing bias and reducing variance (section 3.1, Bühlmann et al., 2014) and (2) data-driven model selection violates key tenets of statistical inference (e.g., Hurvich \& Tsai, 1990; Leeb \& Pötscher, 2005). This is especially relevant for network researchers because prediction is secondary to inference: liberal use of regularization, due to an exaggerated worry of overfitting, unnecessarily compromises statistical inference.

This work also fits within emerging traditions focused on improving methodological practice in psychological science. We offer two further thoughts. First, almost all applied papers justify using lasso by stating it reduces spurious associations and/or mitigates overfitting. The former is incorrect and the latter is vague, with neither providing sufficient justification. Because these statements are widespread and recycled between articles, this is perhaps indicative of cargo cult statistics, that is, "the ritualistic miming of statistics rather than conscientious practice" (p. 40, Stark \& Saltelli, 2018). For possible solutions, we refer interested readers to Stark and Saltelli (2018). Second, we worry that there has been an unforeseen side effect of the "credibility revolution" (Vazire, 2018). With renewed attention to methodology, particular statistical approaches and not their implementation have been unfairly targeted (e.g., the $p$-value). This could contribute to eagerly adopting alternative approaches, say, $\ell_{1}$-regularization, without fully considering their limitations (i.e., are we willing to forego inference?). But as this work demonstrated, the grass is not always greener on the other side of the methodological fence.

\section{Explanation vs. Prediction?}

Our results further suggest that explanation is not the antithesis to prediction. That is, at least in the network literature, there is no apparent need to choose one over the other (Yarkoni \& Westfall, 2017). This has far reaching implications. Predictive models are typically selected based on datadriven procedures, which then, as we stated above, present challenges for inference. On the other hand, our simple approach is based on a valid use of significance testing. We did not select a model first and then seek to make inference. Rather, the network is constructed completely with hypothesis tests. Then, given that conditional dependence structure, a constrained precision matrix can be used for computing predictability if desired. For multiple regression, this was shown to provide more than competitive performance, and, when considering the entire precision matrix, the predictions were typically more accurate than Glasso ${ }_{E B I C}$. This demonstrates that it is not necessary to sacrifice inference to achieve adequate predictive accuracy or to mitigate overfitting.

\section{Limitations}

There are some notable limitations. First, we only considered $\ell_{1}$-regularization for directly estimating the precision matrix. There are alternative forms of regularization that could improve predictive performance (e.g., non-convex penalties, Kim, Lee, \& Kwon, 2018). For large $p / n$ ratios (with small $n$ ) it could be advantageous to employ $\ell_{2}$ -

\footnotetext{
${ }^{4}$ Selecting the true model with probability 1 as $n \rightarrow \infty$.
} 
regularization (Ha \& Sun, 2014; Kuismin, Kemppainen, \& Sillanpää, 2017; Van Wieringen \& Peeters, 2016). On the one hand, the ridge penalty does not push values all the way to zero and thus overcomes the issue of Glasso ${ }_{E B I C}$ selecting empty networks (see Experiment 1). On the other hand, determining the edge set is a key aspect of GGMs and penalization again compromises inference. For completeness, we have provided an example of $\ell_{2}$-regularization in the Appendix ( $\ell_{2}$-Regularization).

Second, there are also approaches that use multiple regression to estimate the network (e.g., Haslbeck \& Waldorp, 2015). We did not consider these, because they do not directly provide an estimate of the precision matrix. But they could reduce test error. For example, one could use automated procedures to select a nonregularized or regularized model based on predictive accuracy or even use the Akaike information criterion (AIC) in place of EBIC. There is a downside. This would compromise the first desideratum of a low error rate, as AIC, which is asymptotically equivalent to LOOCV (Stone, 1977), has a relatively high false positive rate (Dziak, Coffman, Lanza, Li, \& Jermiin, 2019). Furthermore, the quandary of whether it is worth sacrificing inference (due to data-driven model selection) would resurfacethere is no free lunch.

Third, we did not consider alternative motivations for using $\ell_{1}$-regularization. In fact, in the SEM literature, the low false negative rate has been argued to be one key advantage of lasso (Jacobucci et al., 2019). However, it should be noted that reducing the false negative rate can also be achieved in a frequentist framework by simply increasing the alpha level. This seems to be much more straightforward than adopting lasso (e.g., Figure 6). Furthermore, when the rubber meets the road (i.e., time for inference) a valid $p$-value will need to be computed. And, in fact, most of the recent methods for lasso (1) avoid data-driven model selection by fixing the tuning parameter (as implemented in Belloni, Chernozhukov, \& Wang, 2011; Liu \& Wang, 2017; T. Wang et al., 2016); and (2) debias (or "de-sparsify") the estimates such that the model is no longer sparse (Janková \& van de Geer, 2017; Javanmard \& Montanari, 2013, 2015) and then compute confidence intervals for inference. These methods can offer benefits, when, say, $p=100$ and $n=200$ (see Table 1 in Jankova $\&$ van de Geer, 2018). But note this is a rare situation and unnecessary otherwise.

Fourth, we did not consider parameter estimation. This is commonly used to discuss the bias-variance tradeoff (e.g., Figure 1 in Jacobucci et al., 2019). We think this is another source of confusion surrounding overfitting, that is, reducing variance in predictions and an estimator is not same. In our motivating example, the parameters estimates themselves were quite inaccurate (huge MSE). On the other hand, test error was nearly minimized and variance $\left(\mathrm{U}_{v}\right)$ also approached zero (Figure 2, panels B and C). Furthermore, trad- ing in variance for bias is what comprises the sampling distribution of lasso-based estimates. More recent regularization approaches aim to have (1) unbiased estimates (Fan \& $\mathrm{Li}$, 2001; Kim et al., 2018) and (2) the same sampling variance as ordinary least squares estimators (p. 2, Y. Wang \& Zhu, 2016). Investigating these approaches for low-dimensional settings is an interesting future direction.

\section{Recommendations}

We recommend that researchers use nonregularized methods with classical (or Bayesian, Williams \& Mulder, 2019) hypothesis testing. This is implemented in the R packages qgraph (Epskamp et al., 2012), psychonetrics (Epskamp, 2020), and our package GGMnonreg. In our experience, researchers do in fact want to make inference about effects (i.e., edges) and this is not possible with data-driven approaches. Furthermore, because the advantage of those approaches is fleeting, it makes sense to use inferential methods from the start. This also allows for seamless integration of more focused theoretical predictions (Haslbeck, Ryan, Robinaugh, Waldorp, \& Borsboom, 2019), say, testing hypothesized bridge symptoms in psychopathology networks (Castro et al., 2019; Jones, Ma, \& McNally, 2019).

There are some important aspects of our method that must be followed to ensure inference is not compromised. First, choosing an alpha level based on predictive accuracy cannot be done. Rather, researchers must carefully consider the tradeoff that comes from choosing a desired error rate and predictive accuracy prior to estimating the network structure. Figure 6 can be used to inform this decision, for example, by noting the balance between minimizing prediction error and limiting spurious associations for different sample sizes. Second, we again note that the constrained precision matrix can be used for prediction but it would be incorrect to make inference from the estimates. Statistical inference (e.g., computing $p$-values) must be made from the hypothesis tests used to construct the adjacency matrix in (11).

\section{Conclusion}

This work demonstrated that overfitting has been exaggerated in the network literature. We hope this eases methodological anxiety for researchers unfamiliar with the finer details of regularization, prediction, and inference. The ideas discussed in this work provide a foundation from which to begin using nonregularized estimation in combination with classical hypothesis testing. To facilitate this shift, we have implemented the nonregularized approach for predictability in R package GGMnonreg.

\section{References}

Babyak, M. A. (2004). What you see may not be what you get: A brief, nontechnical introduction to overfitting in regression- 
type models. Psychosomatic Medicine, 66(3), 411-421. doi: 10.1097/01.psy.0000127692.23278.a9

Bagby, R. M., Parker, J. D., \& Taylor, G. J. (1994). The twentyitem Toronto Alexithymia scale-I. Item selection and crossvalidation of the factor structure. Journal of Psychosomatic Research, 38(1), 23-32. doi: 10.1016/0022-3999(94)90005 $-1$

Belloni, A., Chernozhukov, V., \& Wang, L. (2011). Square-root lasso: Pivotal recovery of sparse signals via conic programming. Biometrika, 98(4), 791-806. doi: 10.1093/biomet/ asr043

Bento, A. R., Salvação, N., \& Guedes Soares, C. (2018). Validation of a wave forecast system for Galway Bay. Journal of Operational Oceanography, 11(2), 112-124. doi: 10.1080/1755876X.2018.1470454

Berk, R., Brown, L., Buja, A., Zhang, K., \& Zhao, L. (2013). Valid post-selection inference. Annals of Statistics, 41(2), 802837. doi: 10.1214/12-AOS1077

Berk, R., Brown, L., \& Zhao, L. (2010). Statistical inference after model selection. Journal of Quantitative Criminology, 26(2), 217-236.

Bertsimas, D., King, A., \& Mazumder, R. (2016). Best subset selection via a modern optimization lens. Annals of Statistics, 44(2), 813-852. doi: 10.1214/15-AOS1388

Bien, J., \& Tibshirani, R. J. (2011). Sparse estimation of a covariance matrix. Biometrika, 98(4), 807-820. doi: 10.1093/ biomet/asr054

Blanken, T. F., Van Der Zweerde, T., Van Straten, A., Van Someren, E. J., Borsboom, D., \& Lancee, J. (2019). Introducing Network Intervention Analysis to Investigate Sequential, Symptom-Specific Treatment Effects: A Demonstration in Co-Occurring Insomnia and Depression. Psychotherapy and Psychosomatics, 88(1), 52-54. doi: 10.1159/000495045

Borsboom, D. (2017). A network theory of mental disorders. World Psychiatry, 16(1), 5-13. doi: 10.1002/wps.20375

Borsboom, D., \& Cramer, A. O. (2013). Network Analysis: An Integrative Approach to the Structure of Psychopathology. Annual Review of Clinical Psychology, 9(1), 91-121. doi: 10.1146/annurev-clinpsy-050212-185608

Briganti, G., Kempenaers, C., Braun, S., Fried, E. I., \& Linkowski, P. (2018). Network analysis of empathy items from the interpersonal reactivity index in 1973 young adults. Psychiatry Research, 265, 87-92. doi: 10.1016/j.psychres.2018.03.082

Briganti, G., \& Linkowski, P. (2019). Network Approach to Items and Domains From the Toronto Alexithymia Scale. Psychological Reports, 0033294119889586. doi: 10.1177/ 0033294119889586

Brusco, M. J., Voorhees, C. M., Calantone, R. J., Brady, M. K., \& Steinley, D. (2019). Integrating linear discriminant analysis, polynomial basis expansion, and genetic search for twogroup classification. Communications in Statistics: Simulation and Computation, 48(6), 1623-1636. doi: 10.1080/ 03610918.2017.1419262

Bühlmann, P., Kalisch, M., \& Meier, L. (2014). High-Dimensional Statistics with a View Toward Applications in Biology. Annual Review of Statistics and Its Application, 1(1), 255-278. doi: 10.1146/annurev-statistics-022513-115545

Castro, D., Ferreira, F., de Castro, I., Rodrigues, A. R., Correia, M.,
Ribeiro, J., \& Ferreira, T. B. (2019). The Differential Role of Central and Bridge Symptoms in Deactivating Psychopathological Networks. Frontiers in Psychology, 10, 2448. doi: 10.3389/fpsyg.2019.02448

Chaudhuri, S., Drton, M., \& Richardson, T. (2007). Estimation of a covariance matrix with zeros. Biometrika, 94(1).

Christensen, A. P., \& Golino, H. (2019). Estimating the stability of the number of factors via Bootstrap Exploratory Graph Analysis: A tutorial. PsyArXiv. doi: 10.31234/OSF.IO/9DEAY

Clements, M., \& Hendry, D. (1998). Forecasting economic time series. Cambridge: Cambridge University Press.

Costantini, G., Epskamp, S., Borsboom, D., Perugini, M., Mõttus, R., Waldorp, L. J., \& Cramer, A. O. (2015). State of the aRt personality research: A tutorial on network analysis of personality data in R. Journal of Research in Personality, 54, 13-29. doi: 10.1016/j.jrp.2014.07.003

Danilov, D., \& Magnus, J. R. (2004). On the harm that ignoring pretesting can cause. Journal of Econometrics, 122(1), 2746. doi: 10.1016/j.jeconom.2003.10.018

Davis, M. H. (1983). A Multidimensional Approach to Individual Differences in Empathy. JSAS Catalog of Selected Documents in Psychology.

Di Pierro, R., Costantini, G., Benzi, I. M. A., Madeddu, F., \& Preti, E. (2019). Grandiose and entitled, but still fragile: A network analysis of pathological narcissistic traits. Personality and Individual Differences, 140, 15-20. doi: 10.1016/j.paid.2018.04.003

Drton, M., \& Perlman, M. D. (2004). Model selection for Gaussian concentration graphs. Biometrika, 91(3), 591-602. doi: 10.1093/biomet/91.3.591

Dziak, J. J., Coffman, D. L., Lanza, S. T., Li, R., \& Jermiin, L. S. (2019). Sensitivity and specificity of information criteria. Briefings in Bioinformatics, 00(March), 1-13. doi: 10.1093/bib/bbz016

Emmert-Streib, F., Tripathi, S., \& Dehmer, M. (2019). Constrained Covariance Matrices With a Biologically Realistic Structure: Comparison of Methods for Generating High-Dimensional Gaussian Graphical Models. Frontiers in Applied Mathematics and Statistics, 5, 17. doi: 10.3389/fams.2019.00017

Epskamp, S. (2020). psychonetrics: Structural Equation Modeling and Confirmatory Network Analysis. Retrieved from https://cran.r-project.org/package= psychonetrics

Epskamp, S., Cramer, A. O. J., Waldorp, L. J., Schmittmann, V. D., \& Borsboom, D. (2012). qgraph: Network Visualizations of Relationships in Psychometric Data. Journal of Statistical Software, 48(4). doi: 10.18637/jss.v048.i04

Epskamp, S., Waldorp, L. J., Mottus, R., \& Borsboom, D. (2018). The Gaussian Graphical Model in Cross-Sectional and TimeSeries Data. Multivariate Behavioral Research, 53(4), 453480. doi: 10.1080/00273171.2018.1454823

Fan, J., \& Li, R. (2001). Variable Selection via Nonconcave Penalized Likelihood and its Oracle Properties. Journal of the American Statistical Association, 96(456), 1348-1360. doi: 10.1198/016214501753382273

Fitzgerald, E., \& Akintoye, A. (1995). The accuracy and optimal linear correction of UK construction tender price index forecasts. Construction Management and Economics, 13(6), 
493-500. doi: 10.1080/01446199500000057

Fried, E. I., \& Cramer, A. O. (2017). Moving Forward: Challenges and Directions for Psychopathological Network Theory and Methodology. Perspectives on Psychological Science, 12(6), 999-1020. doi: 10.1177/1745691617705892

Gao, X., Pu, D. Q., Wu, Y., \& Xu, H. (2009). Tuning parameter selection for penalized likelihood estimation of inverse covariance matrix. arXiv. doi: 10.5705/ss.2009.210

Gelman, A., Hwang, J., \& Vehtari, A. (2014). Understanding predictive information criteria for Bayesian models. Statistics and Computing, 24(6), 997-1016. doi: 10.1007/s11222-013 $-9416-2$

Goodwin, P. (1997). Adjusting judgemental extrapolations using Theil's method and discounted weighted regression. Journal of Forecasting, 16(1), 37-46. doi: 10.1002/(SICI)1099 -131X(199701)16:1<37::AID-FOR647>3.0.CO;2-T

Gronau, Q. F., \& Wagenmakers, E.-J. (2018). Limitations of Bayesian Leave-One-Out Cross-Validation for Model Selection. PsyArXiv. doi: 10.17605/OSF.IO/AT7CX

Ha, M. J., \& Sun, W. (2014). Partial correlation matrix estimation using ridge penalty followed by thresholding and reestimation. Biometrics, 70(3), 765-773. doi: 10.1111/ biom. 12186

Haslbeck, J. M., \& Fried, E. I. (2017). How predictable are symptoms in psychopathological networks? A reanalysis of 18 published datasets. Psychological Medicine, 47(16), 22672276. doi: 10.1017/S0033291717001258

Haslbeck, J. M., Ryan, O., Robinaugh, D., Waldorp, L., \& Borsboom, D. (2019). Modeling Psychopathology: From Data Models to Formal Theories. PsyArXiv. doi: 10.31234/ OSF.IO/JGM7F

Haslbeck, J. M., \& Waldorp, L. J. (2015). MGM: Estimating Time-Varying Mixed Graphical Models in HighDimensional Data.

Haslbeck, J. M., \& Waldorp, L. J. (2018). How well do network models predict observations? On the importance of predictability in network models. Behavior Research Methods, 50(2), 853-861. doi: 10.3758/s13428-017-0910-x

Hastie, T., Tibshirani, R., \& Friedman, J. (2008). The Elements of Statistical Learning: Data Mining, Inference, and Prediction (2nd ed.). New York: Springer.

Hastie, T., Tibshirani, R., \& Tibshirani, R. J. (2017). Extended Comparisons of Best Subset Selection, Forward Stepwise Selection, and the Lasso. arXiv.

Hastie, T., Tibshirani, R., \& Wainwright, M. (2015). Statistical Learning with Sparsity: The Lasso and Generalizations. Boca Raton: CRC Press. doi: 10.1201/b18401-1

Hevey, D. (2018). Network analysis: a brief overview and tutorial. Health Psychology and Behavioral Medicine, 6(1), 301-328. doi: 10.1080/21642850.2018.1521283

Højsgaard, S., Edwards, D., \& Lauritzen, S. (2012). Graphical Models with R. doi: 10.1007/978-1-4614-2299-0

Hurvich, C. M., \& Tsai, C.-L. (1990). The Impact of Model Selection on Inference in Linear Regression. The American Statistician, 44(3), 214. doi: 10.2307/2685338

Hyndman, R. J., \& Athanasopoulos, G. (2018). Forecasting: principles and practice (2nd ed.). Melbourne: OTexts.

Jacobucci, R., Brandmaier, A. M., \& Kievit, R. A. (2019). A
Practical Guide to Variable Selection in Structural Equation Modeling by Using Regularized Multiple-Indicators, Multiple-Causes Models. Advances in Methods and Practices in Psychological Science, 2(1), 55-76. doi: 10.1177/ 2515245919826527

Janková, J., \& van de Geer, S. (2017). Honest confidence regions and optimality in high-dimensional precision matrix estimation. Test, 26(1), 143-162. doi: 10.1007/s11749-016-0503 $-5$

Jankova, J., \& van de Geer, S. (2018). Inference in highdimensional graphical models. arxiv, 0, 1-29.

Javanmard, A., \& Montanari, A. (2013). Confidence Intervals and Hypothesis Testing for High-Dimensional Regression. , 121.

Javanmard, A., \& Montanari, A. (2015). De-biasing the Lasso: Optimal Sample Size for Gaussian Designs. arXiv.

Jones, P. J., Heeren, A., \& McNally, R. J. (2017). Commentary: A network theory of mental disorders. Frontiers in Psychology, 8, 1305. doi: 10.3389/fpsyg.2017.01305

Jones, P. J., Ma, R., \& McNally, R. J. (2019). Bridge Centrality: A Network Approach to Understanding Comorbidity. Multivariate Behavioral Research, 1-15. doi: 10.1080/ 00273171.2019 .1614898

Kabaila, P. (1995). The effect of model selection on confidence regions and prediction regions. Econometric Theory, 11(3), 537-549. doi: 10.1017/S0266466600009403

Kim, D., Lee, S., \& Kwon, S. (2018). A unified algorithm for the non-convex penalized estimation: The ncpen package. arxiv.

Krämer, N., Schäfer, J., \& Boulesteix, A.-L. (2009). Regularized estimation of large-scale gene association networks using graphical Gaussian models. BMC Bioinformatics, 10(1), 384. doi: 10.1186/1471-2105-10-384

Kuismin, M., Kemppainen, J., \& Sillanpää, M. (2017). Precision Matrix Estimation With ROPE. Journal of Computational and Graphical Statistics, 26(3), 682-694. doi: 10.1080/10618600.2016.1278002

Kuismin, M., \& Sillanpää, M. (2017). Estimation of covariance and precision matrix, network structure, and a view toward systems biology. Wiley Interdisciplinary Reviews: Computational Statistics, 9(6), 1-13. doi: 10.1002/wics.1415

Kwan, C. C. Y. (2014). A Regression-Based Interpretation of the Inverse of the Sample Covariance Matrix. Spreadsheets in Education, 7(1).

Lauritzen, S. L. (1996). Graphical models (Vol. 17). Clarendon Press.

Ledoit, O., \& Wolf, M. (2004). A well-conditioned estimator for large-dimensional covariance matrices. Journal of Multivariate Analysis, 88(2), 365-411. doi: 10.1016/S0047 -259X(03)00096-4

Lee, J. D., Sun, D. L., Sun, Y., \& Taylor, J. E. (2016). Exact postselection inference, with application to the lasso. Annals of Statistics, 44(3), 907-927. doi: 10.1214/15-AOS1371

Leeb, H., \& Pöscher, B. M. (2006). Can one estimate the conditional distribution of post-model-selection estimators? Annals of Statistics, 34(5), 2554-2591. doi: 10.1214/ 009053606000000821

Leeb, H., \& Pötscher, B. M. (2005). Model selection and inference: 
Facts and fiction. Econometric Theory, 21(1), 21-59. doi: $10.1017 / \mathrm{S} 0266466605050036$

Leeb, H., Pötscher, B. M., \& Ewald, K. (2015). On Various Confidence Intervals Post-Model-Selection. Statistical Science, 30(2), 216-227. doi: 10.1214/14-STS507

Leppä-aho, J., Pensar, J., Roos, T., \& Corander, J. (2017). Learning Gaussian graphical models with fractional marginal pseudolikelihood. International Journal of Approximate Reasoning, 83, 21-42. doi: 10.1016/j.ijar.2017.01.001

Liu, H., \& Wang, L. (2017). TIGER: A tuning-insensitive approach for optimally estimating gaussian graphical models. Electronic Journal of Statistics, 11(1), 241-294. doi: 10.1214/16-EJS1195

Mazumder, R., Radchenko, P., \& Dedieu, A. (2017). Subset Selection with Shrinkage: Sparse Linear Modeling when the SNR is low. arXiv.

Meinshausen, N., \& Bühlmann, P. (2006). High-dimensional graphs and variable selection with the Lasso. Annals of Statistics, 34(3), 1436-1462. doi: 10.1214/ 009053606000000281

Miner, J., \& Mincer, V. (1969). The Evaluation of Economic Forecasts. In J. Mincer (Ed.), Economic forecasts and expectations: Analysis offorecasting behavior and performance (pp. 3-46). New York: National Bureau of Economic Research.

Murphy, A. H. (1988). Skill Scores Based on the Mean Square Error and Their Relationships to the Correlation Coefficient. Monthly Weather Review, 148(3). doi: 10.1175/ 1520-0493(1988)116<2417:SSBOTM > 2.0.CO;2

Revelle, W. (2019). psych: Procedures for Psychological, Psychometric, and Personality Research. Evanston, Illinois. Retrieved from https://cran.r-project.org/package= psych

Ryan, O., Bringmann, L., \& Schuurman, N. K. (2019). The Challenge of Generating Causal Hypotheses Using Network Models. doi: 10.31234/OSF.IO/RYG69

Schäfer, J., \& Strimmer, K. (2005). A Shrinkage Approach to Large-Scale Covariance Matrix Estimation and Implications for Functional Genomics. Statistical Applications in Genetics and Molecular Biology, 4(1). doi: 10.2202/1544-6115 .1175

Solazzo, E., \& Galmarini, S. (2016). Error apportionment for atmospheric chemistry-transport models - A new approach to model evaluation. Atmospheric Chemistry and Physics, 16(10), 6263-6283. doi: 10.5194/acp-16-6263-2016

Solazzo, E., Hogrefe, C., Colette, A., Garcia-Vivanco, M., \& Galmarini, S. (2017). Advanced error diagnostics of the CMAQ and Chimere modelling systems within the AQMEII3 model evaluation framework. Atmospheric Chemistry and Physics, 17(17), 10435-10465. doi: 10.5194/acp-17-10435-2017

Stark, P. B., \& Saltelli, A. (2018). Cargo-cult statistics and scientific crisis. Significance, 15(4), 40-43. doi: 10.1111/ j.1740-9713.2018.01174.x

Stephens, G. (1998). On the Inverse of the Covariance Matrix in Portfolio Analysis. The Journal of Finance, 53(5), 18211827.

Stone, M. (1977). An Asymptotic Equivalence of Choice of Model by Cross-Validation and Akaike's Criterion. Journal of the
Royal Statistical Society: Series B (Methodological), 39(1), 44-47. doi: 10.1111/j.2517-6161.1977.tb01603.x

Taylor, J., \& Tibshirani, R. (2017). Post-selection inference for $\ell 1$-penalized likelihood models. Canadian Journal of Statistics(2015), 1-26. doi: 10.1002/cjs.11313

Taylor, J., \& Tibshirani, R. J. (2015). Statistical learning and selective inference. Proceedings of the National Academy of Sciences, 112(25), 7629-7634.

Theil, H. (1961). Economic forecast and policy. North-Holland.

Van Wieringen, W. N., \& Peeters, C. F. (2016). Ridge estimation of inverse covariance matrices from high-dimensional data. Computational Statistics and Data Analysis, 103, 284-303. doi: 10.1016/j.csda.2016.05.012

Vazire, S. (2018). Implications of the Credibility Revolution for Productivity, Creativity, and Progress. Perspectives on psychological science : a journal of the Association for Psychological Science, 13(4), 411-417. doi: 10.1177/ 1745691617751884

Waldorp, L., Marsman, M., \& Maris, G. (2019). Logistic regression and Ising networks: prediction and estimation when violating lasso assumptions. Behaviormetrika, 46(1), 49-72. doi: 10.1007/s41237-018-0061-0

Wang, T., Ren, Z., Ding, Y., Fang, Z., Sun, Z., MacDonald, M. L., ... Chen, W. (2016). FastGGM: An Efficient Algorithm for the Inference of Gaussian Graphical Model in Biological Networks. PLoS Computational Biology, 12(2), 1-16. doi: 10.1371/journal.pcbi.1004755

Wang, Y., \& Zhu, L. (2016). Variable Selection and Parameter Estimation with the Atan Regularization Method. Journal of Probability and Statistics, 2016, 1-12. doi: 10.1155/2016/ 6495417

Watson, P., \& Teelucksignh. (2008). A Practical Introduction to Econometric Methods: Classical and Modern (1st ed.). Kingston: University of the West Indies Press.

Werner, M., Štulhofer, A., Waldorp, L., \& Jurin, T. (2018). A Network Approach to Hypersexuality: Insights and Clinical Implications. Journal of Sexual Medicine, 15(3), 410-415. doi: 10.1016/j.jsxm.2018.01.009

Williams, D. R., \& Mulder, J. (2019). Bayesian Hypothesis Testing for Gaussian Graphical Models: Conditional Independence and Order Constraints. PsyArXiv. doi: 10.31234/osf.io/ ypxd8

Williams, D. R., \& Rast, P. (2019). Back to the basics: Rethinking partial correlation network methodology. British Journal of Mathematical and Statistical Psychology. doi: 10.1111/bmsp. 12173

Williams, D. R., Rhemtulla, M., Wysocki, A. C., \& Rast, P. (2019). On Nonregularized Estimation of Psychological Networks. Multivariate Behavioral Research, 54(5), 1-23. doi: 10.1080/00273171.2019.1575716

Wysocki, A. C., \& Rhemtulla, M. (2019). On Penalty Parameter Selection for Estimating Network Models. Multivariate Behavioral Research, 1-15. doi: 10.1080/00273171.2019 .1672516

Yang, Y. (2005). Can the strengths of AIC and BIC be shared? A conflict between model indentification and regression estimation. Biometrika, 92(4).

Yarkoni, T., \& Westfall, J. (2017). Choosing Prediction Over Ex- 
planation in Psychology: Lessons From Machine Learning. Perspectives on Psychological Science, 12(6), 1100-1122. doi: $10.1177 / 1745691617693393$

Yuan, M. (2010). High Dimensional Inverse Covariance Matrix Estimation via Linear Programming. Journal of Machine Learning Research, 11, 2261-2286.

Zhang, R., Ren, Z., \& Chen, W. (2018). SILGGM: An extensive R package for efficient statistical inference in large-scale gene networks. PLoS Computational Biology, 14, e1006369. doi: 10.1371/journal.pcbi.1006369

Zhao, P., \& Yu, B. (2006). On Model Selection Consistency of Lasso. The Journal of Machine Learning Research, 7, 25412563. doi: 10.1109/TIT.2006.883611
Appendix A

Estimating a Precision Matrix with Zeros

Algorithm 1 Description of the HTF algorithm (pp. 631634, Hastie et al., 2008)

$\mathbf{S}$ is a $p \times p$ covariance matrix

$\mathbf{A}$ is a $p \times p$ adjacency matrix

Initialize $\mathbf{W}=\mathbf{S}$

repeat

For nodes $j=1, \ldots, p$

(a) Partition the matrices: $\mathbf{W}_{11}=\mathbf{W}_{-j,-j}$ and $\mathbf{s}_{12}=\mathbf{S}_{j,-j}$

(b) Obtain the row indices that define the neighborhood, $n e_{j}$, in $\mathbf{A}_{j,-j}$ (Equation 11)

(c) Subset the matrices according to the neighborhood, that is, $\mathbf{W}_{11}^{n e_{j}}$ and $\mathbf{s}_{12}^{n e_{j}}$.

Note: The rows and columns of $\mathbf{W}_{11}$ are subsetted according to $n e_{j}$, resulting in the sample covariance matrix with respect to $\mathbf{A}_{j,-j}$.

(d) Initialize a $(p-1) \times 1$ vector of zeroes, $\boldsymbol{\beta}$, to gather the regression coefficients.

(e) Compute the regression coefficients: $\hat{\boldsymbol{\beta}}^{n e_{j}}=$ $\mathbf{W}_{11}^{n e_{j}^{-1}} \mathbf{s}_{12}^{n e_{j}}$, where the coefficients are filled into the positions of 1's that were obtained from step (b).

Note: This corresponds to solving for $\hat{\boldsymbol{\beta}}^{n e_{j}}$ with subsets of $\mathbf{Y}: \hat{\boldsymbol{\beta}}^{n e_{j}}=\left(\mathbf{Y}_{n e_{j}}^{\prime} \mathbf{Y}_{n e_{j}}\right)^{-1} \mathbf{Y}_{n e_{j}}^{\prime} \mathbf{Y}_{j}$, where $\mathbf{W}_{11}^{-1}=\left(\mathbf{Y}_{n e_{j}}^{\prime} \mathbf{Y}_{n e_{j}}\right)^{-1} \cdot(n-1)$ and $\mathbf{S}_{11}=$ $\mathbf{Y}_{n e_{j}}^{\prime} \mathbf{Y}_{j} /(n-1)$. Here $\mathbf{Y}_{n e_{j}}$ denotes the subset of columns in $\mathbf{Y}$ that correspond to the neighborhood of $\mathbf{Y}_{j}$.

(f) Update $\mathbf{W}: \mathbf{W}_{j,-j}=\mathbf{W}_{-j, j}=\mathbf{W}_{11} \hat{\boldsymbol{\beta}}$

end for

if $\max _{i j}\left(\mathbf{W}-\mathbf{W}_{\text {previous }}\right)<$ tolerance

end repeat

return $\hat{\mathbf{\Theta}}=\mathbf{W}^{-1}$

else

$\mathbf{W}_{\text {previous }}=\mathbf{W}$

In this algorithm, $\mathbf{W}_{11}$ is the sample covariance matrix, $\mathbf{S}$, but excluding the $j$ th row and column. Further, $\mathbf{s}_{12}$ is the $j$ th row of $\mathbf{S}$, excluding the $j$ th column. In step (c), an additional subset of each is then obtained according to the neighborhood of the $j$ th node, corresponding to those relations determined to be significantly different than zero (i.e., the location of 1's $\mathbf{A}_{j,-j}$ ).

Appendix B 


\section{Rationale}

$\ell_{2}$-Regularization

In full disclosure, we were hoping to provide a clear picture of where $\ell_{1}$-regularization provided notable benefits. We struggled to find such examples that would also be representative of the network literature. We thus repeated Experiment 3 with $\ell_{2}$-regularization (i.e., ridge) instead of $\ell_{1}$-regularization. The aim was to explore whether $\ell_{2}$-regularization overcomes the issues of Glasso ${ }_{E B I C}$ selecting empty networks and having large test error for the largest $p / n$ ratios. In this example, the training data sizes ranged from 50 to 200 (in increments of 10). We estimated the ridge-based precision matrix using the approach outlined in Ha and Sun (2014). ${ }^{5}$ This particular method estimates the optimal shrinkage parameter analytically (see Equations 4-7 in Schäfer \& Strimmer, 2005).

Results. The results (see Figure B1) show that the ridge approach not only overcomes the issues of the lasso at larger $p / n$ ratios, but provided more than competitive predictive performance when compared to Nonreg $_{N H S T}$ and the full model. This is seen, for example, in the BFI dataset, where the ridge approach outperformed both nonregularized methods at all sample sizes. However, the methods ultimately converged to be quite similar. A similar pattern can be observed across the IRI and TAS datasets. Note that there are many partial correlations in each network (BFI: 300, IRI: 378, TAS: 190). This again makes clear that nonregularized estimation provides competitive performance even when there are more partial correlations than observations (the number of nodes is what matters). Together, while there are situations where regularization may be employed for a gain in predictive accuracy, one must be cognizant that this sacrifices two desirable goals in network analysis: (1) edge selection cannot be performed because the $\ell_{2}$-penalty does not push any estimates to zero; and (2) inference is compromised by introducing bias from the shrinkage penalty.
${ }^{5}$ This was implemented with the R package GGMridge. 
Full Nonreg $_{N H S T}=$ Ridge
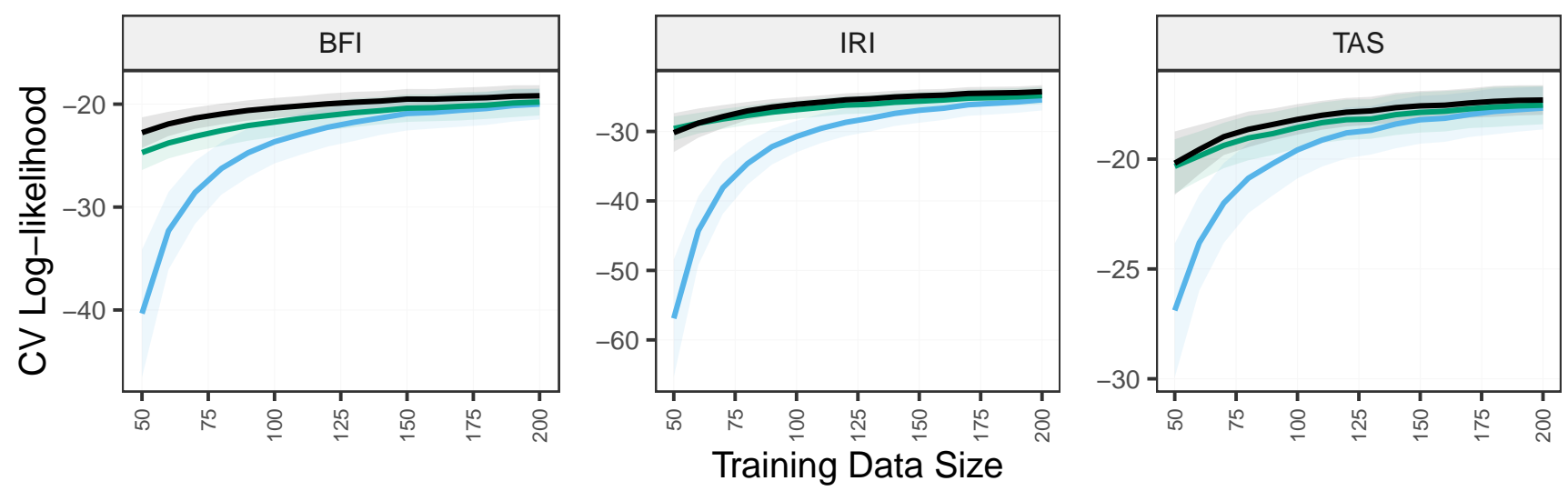

Figure B1. The $y$-axis is the cross-validated log-likelihood (Equation 12). This provides a measure of global predictive accuracy for the network. Larger values indicate superior performance (i.e., maximizing the likelihood). BFI $(p=25)$, IRI $(p=28)$, and TAS $(p=20)$. 